Modern Physics Letters B

(c) World Scientific Publishing Company

\title{
Defect Engineering of 2D Monatomic-layer Materials
}

\author{
Qing Peng \\ Department of Mechanical, Aerospace and Nuclear Engineering, \\ Rensselaer Polytechnic Institute, Troy, NY 12180, U.S.A. \\ qpeng.org@gmail.com \\ Jared Crean \\ Department of Mechanical, Aerospace and Nuclear Engineering, \\ Rensselaer Polytechnic Institute, Troy, NY 12180, U.S.A.
}

Albert K. Dearden

Department of Physics, Applied Physics, and Astronomy, Rensselaer Polytechnic Institute, Troy, NY 12180, U.S.A.

Chen Huang

Theoretical Division, Los Alamos National Laboratory, Los Alamos, New Mexico 87545, U.S.A

Xiaodong Wen

Theoretical Division, Los Alamos National Laboratory, Los Alamos, New Mexico 87545, U.S.A

Stéphane P. A. Bordas

Cardiff University, School of Engineering, Queen's Buildings, The Parade, Cardiff, CF24 3 AA Wales, UK

Suvranu De

Department of Mechanical, Aerospace and Nuclear Engineering, Rensselaer Polytechnic Institute, Troy, NY 12180, U.S.A.

Received (1 June 2013)

\begin{abstract}
Atomic-thick monolayer two-dimensional materials present advantageous properties compared to their bulk counterparts. The properties and behavior of these monolayers can be modified by introducing defects namely defect engineering. We review a group of common 2D crystals, including graphene, graphyne, graphdiyne, graph $n$-yne, silicene, germanene, hexagonal boron nitride monolayers, and $\mathrm{MoS}_{2}$ monolayers, focusing on the effect of the defect engineering on these two-dimensional monolayer materials. Defect engineering leads to the discovery of potentially exotic properties that make the field of two-dimensional crystals fertile for future investigations and emerging technological applications with precisely tailored properties.
\end{abstract}

Keywords: Defect engineering; 2D materials; Monatomic layer. 


\section{INTRODUCTION}

Due to its unique physical properties, graphene has attracted tremendous attention. In the mean time, the discovery of the graphene signaled the start of a race exploring atomically thick two-dimentional (2D) materials ${ }^{1}$. Because of quantum confinement in the third dimension, these 2D materials demonstrated distinct properties from their bulk counterparts. There are many reviews investigating these $2 \mathrm{D}$ materials ${ }^{2,3}$, however the effects of defects and defect engineering have been less studied and reviewed. In this paper we review recent studies of defects in such $2 \mathrm{D}$ one atom thick materials.

Crystals are inherently imperfect, and contain defects. For example, vacancies and self-interstitial atoms can statistically be formed and annihilated by thermodynamics. However, defects are integral to the semi-conductor industry, as with the addition of just one dopant atom per hundred million host atoms significantly alters the electronic properties of the host material. This phenomenon has been used for years to improve nano-semi-conductors and thus facilitate miniaturisation of electronic devices. Each dopant can then be seen as a kind of atomic point defect. As such, defects in 2D crystals are an attractive target because their two dimensional nature makes it easier to add, remove, or move atoms to alter their electronic properties, in a process known as self-doping. Defects can also be patterned into extended structures to give entirely new properties. Theorists have proposed that such extended defects could be used to modify the electronic properties of materials.

Such defects and dopants have the possibility of modifying the magnetic properties within these structures. Defects that result in zigzag edges within structures give rise to magnetism that would be useful for spintronic devices. These zigzag edges can exist in structures such as graphene nanoribbons as a terminating edge state, however point defects such as a single or small cluster of atomic vacancies may also produce zigzag states within graphene. Dopant atoms within graphene may also induce magnetism that can be modulated by the concentration of dopants. Furthermore, graphene-like systems can also experience magnetism due to defects. Such materials could prove useful for device applications involving spin control in situations where simple graphene would be unviable.

There are many graphene-like 2D materials being explored ${ }^{2,3,4,5,6,7,8}$. In order to keep this review concise, we restrict ourselves to "popular" $2 \mathrm{D}$ materials, such as graphene, graphyne and its families, monolayer $h$-BN, silicene, germanene, and monolayer $\mathrm{MoS}_{2}$.

The graphyne family consists of variations on graphene, formed by introducing acetylenic linkages within the hexagonal lattice of graphene. Several forms of graphene have been predicted by Baughman and Eckardt, with varying concentrations of acetylenic linkages ${ }^{9}$, leading to new properties not seen in graphene. The mechanical, electronic, and thermal properties have been investigated using computational techniques including Molecular Dynamics and Density Functional Theory, but also continuum methods. One possibility of considerable interest is 
semi-conduction, which led to studies of charge carrier mobility and band gap tuning. The ability to tune the band gap would allow for the customization of materials to a particular application, potentially leading to the creation of more effective devices. Various means of altering these properties, including slightly altering the lattice structures, introducing vacancies, and adsorbing other types of atoms onto the sheets of the different graphynes, will be discussed in addition to the mechanical differences between the various forms of graphyne. Graphyne has yet to be synthesized, so computational studies are the primary methodology for the studies discussed in this section.

Hexagonal boron nitride is an analogue of graphene, in that it is formed from the same hexagonal lattice, however it consists of an alternating pattern of boron and nitrogen atoms rather than purely carbon atoms. Experimental as well as computational methods have produced results regarding the mechanical, electronic, and thermal properties of this material. Additionally, because of the similar lattice structure of hexagonal boron nitride to graphene, hybrid structures have been investigated that combine the two materials into a single crystal structure. While the mechanical properties are impressive, significant effort has been applied to the investigation of the electronic and magnetic properties. The effects of vacancies and doping on the band gap, as well as adsorption of other atoms, have been studied and will be discussed. Synthesis of multi-layer hexagonal boron nitride has been accomplished, and some experimental results are discussed in addition to the results of computational studies, although comparisons are limited because a single layer of the material has not been experimentally examined yet.

As carbon is immediately next to silicon on the periodic table, a sensible question to ask was whether similarly revolutionary properties as those obtained by creating one atom thick layers of carbon could be obtained from the creation of similar layers made of silicon. The result was silicene, which is the thinnest possible form of silicon and can be considered as being to silicon what graphene is to carbon.

Silicene has been studied with interest, possibly because it could be more compatible with existing silicon-based electronics than graphene. Its combination with germanene, the graphene-analogue of Germanium could provide a transition semiconducting material until graphene-based semi-conductors become more efficient.

Molybdenum disulfide $\left(\mathrm{MoS}_{2}\right)$ monolayers have received enormous attention due to their striking optical, electronic, and mechanic properties ${ }^{10,11}$. Monolayer $\mathrm{MoS}_{2}$ has a direct optical gap of $1.8 \mathrm{eV}^{12,13,14}$. The presence of this band gap makes monolayer $\mathrm{MoS}_{2}$ interesting for applications in nanoelectronics where it allows for the fabrication of transistors with low power dissipation and current on/off ratios. The applications of $\mathrm{MoS}_{2}$ also includes field-effect transistors $(\mathrm{FET})^{15,16}$, valleytronics ${ }^{17,18,19,20,21,22}$, phototransistors ${ }^{23,24}$, gas sensors ${ }^{25}$, catalytic hydrodesulfonization ${ }^{26,27}$, hydrogen evolution ${ }^{28,29}$, photoelectrochemcial hydrogen production ${ }^{30,31,32,33}$, small-signal amplifiers ${ }^{34}$, solid lubricants ${ }^{35}$, and nonvolatile memory cells ${ }^{36}$. In addition, monolayer $\mathrm{MoS}_{2}$ has promising applications in flexible electronics where it would combine high performance with low cost. 
The rest of the paper is organized as follows. Section II focuses on graphene, Section III on graphyne, graphdi-yne, and graph $n$-yne. Section IV addresses $h$-BN monolayers whilst Section V discusses silicene and germanene. Section VI, focusing on $\mathrm{MoS}_{2}$ monolayers, is followed by conclusions in Section VII.

\section{Graphene}

\subsection{Magnetic Properties}

Since its discovery ${ }^{1}$, graphene along with its derivative structures, have been shown to have a number of interesting properties. Here, we examine the recent studies on the magnetic properties of graphene. Ordinarily, a pristine graphene sheet is a two dimensional crystal composed of two sublattices of carbon atoms, lattice A and lattice $\mathrm{B}$, that displays no net magnetic moment. However, magnetism may be induced through engineering the shape of the graphene sheets into other forms, such as nanoribbons or zero dimensional structures. Additionally, magnetism may be induced in graphene by introducing defects such as vacancies in specified locations as well as through doping. By being able to precisely control the magnetic properties of graphene, a wide range of applications is possible.

\subsubsection{Magnetism due to Edge Shape}

According to the Hubbard model and benzenoid graph theory ${ }^{37,38}$, an imbalance between the A and B lattice sites in a bipartite lattice will result in a magnetic ground state. This can be seen in cases where graphene has been cut into structures with zigzag edge shapes ${ }^{39,40,41,42}$. Examining Figure 1, along a single zigzag direction there is an imbalance of lattice sites. Note however, that along the armchair direction there is no imbalance, thus there is no induced magnetism for graphene structures containing only armchair edges. Furthermore while there exists a net magnetization, it is however not uniform throughout the structure. Moving away from the source of the magnetism, in this case the zigzag edges, results in a decay of the magnetization on each carbon atom until, for a sufficiently large distance, there is no longer an individual magnetization on the carbon atoms and that region of the structure returns to the usual nonmagnetic state.

The magnetism resulting from edge states in structures such as ZGNRs also plays an important role in transport properties. In a brief report which compares a pristine and a Klein defect ZGNR ${ }^{43}$, it is found that with the presence of an external gate, the Klein edge defects result in a transition from pure spin current to a spin degenerate charge current that is close to the Fermi energy. Furthermore, with the inclusion of a Klein defect, the ZGNR exhibits a net magnetization with a spin split band gap. Using a gate-injected charge density that was obtained self consistently along with conductance calculations performed within Landauer's formalism, it was found that an external gate could tune the band gap in both pristine ZGNRs and Klein edge defect ZGNRs. For pristine ZGNRs, the external gate causes a switching 


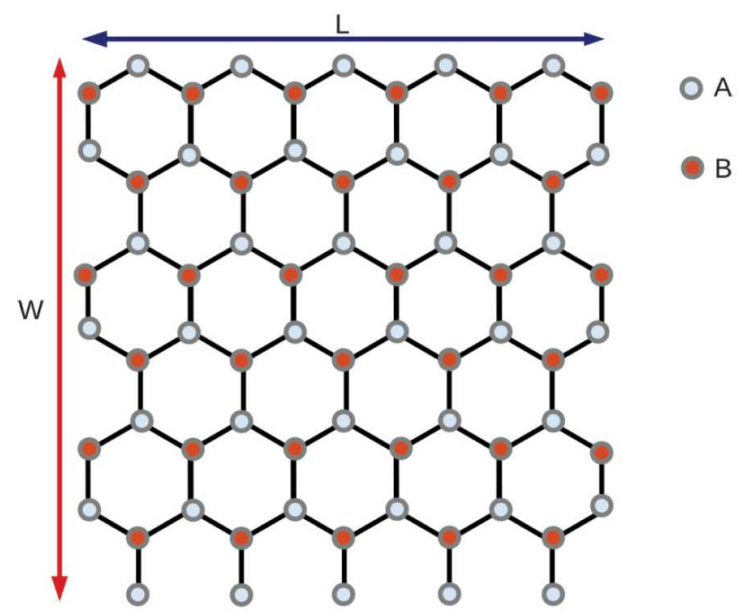

Fig. 1. (Color online) Example of the A and B lattice configuration with a top zigzag edge. Reproduced with permission ${ }^{39}$. Copyright 2012 American Physical Society.

on of charge current with the up and down spin electrons being equivalent. However, in the case of the Klein defect ZGNRs, within approximately $1.0 \mathrm{~V}$, only the majority of the spin, either up or down, transmits. Beyond $1.0 \mathrm{~V}$, the system turns nonmagnetic and both spins will have identical transmission. This indicates that it is possible to tune the current in ZGNRs from a spin polarized to an unpolarized state by simply modulating the external gate.

\subsubsection{Magnetism due to Vacancies}

Aside from edge shape, disruption of the balance between A and B lattice sites through vacancy defects also induces magnetism. Theoretical studies show that vacancies in graphene sheets ${ }^{44}$ as well as in graphene nanoribbons ${ }^{45,46}$ (GNRs) induce magnetism whose magnitude is determined by the number and size of the defects. It is shown that vacancies created in one of the two lattices result in a net magnetic moment, however with vacancies corresponding to both lattices, the net magnetic moment disappears. It is indicated that each dangling orbital that appears as a result of the vacancy contributes $1.0 \mu_{B}$, and any remaining magnetic moment corresponds to the imbalance in spin polarized $\pi$ orbitals. They go on to show that with large enough vacancies or appropriate configurations, there may exist a zero net magnetic moment despite the presence of zigzag edges. This is not contradictory for the previous section because while zigzag edges exist, they are offset by armchair components that result in a balance of the A and B lattices. Thus, while locally there is a magnetic moment on each atom that represents a ferromagnetic ordering, there exists another ferromagnetic ordering elsewhere in the structures that are of opposite spin. Furthermore, in a recent experiment ${ }^{47}$, nanopores were created in 
graphene through chemical vapor deposition that resulted in ferro-magnetism, with a saturation magnetization of $0.04 \mathrm{emu} / \mathrm{g}$, that was stable at room temperature. The source of the magnetization is from the high density of zigzag edges that were present as a result of the newly created vacancies.

\subsubsection{Magnetism due to Doping}

Another way to induce magnetism in graphene structures is through doping. Through lithium doping ${ }^{48}$, the edges of zigzag graphene nanoribbons (ZGNRs) can be tuned in a non-destructive manner. When lithium is adsorbed onto ZGNRs, there is a strong tendency to bind with the edges of the ZGNR. This is shown by the fact that there is a large energy barrier, roughly $0.6 \mathrm{eV}$, for the lithium atom to move from one edge of a 10-ZGNR to the other. Due to the lithium atom binding only along the edges, it is possible to tune the two edges of a ZGNR individually. By introducing the lithium atom to the structure, the spin polarization is reduced along that edge, allowing for tuning of the magnetization of the ZGNR.

Other forms of doping may be used to alter the magnetics of graphene such as hybrid carbon and boron nitride structures ${ }^{49}$. The advantage of such structures is that the band gap of the resulting composite structure may be modulated by changing the ratio of carbon with boron nitride. By introducing appropriate graphene structures with zigzag edges into a boron nitride layer, magnetism may be induced which can be tuned by changing the size of the graphene segments. It is reported that the resulting composite structures exhibit stabilities similar to that of pure boron nitride sheets.

\subsubsection{Graphene-like Systems}

In addition to graphene or graphene based materials, the magnetics of structures that resemble the hexagonal graphene lattice but consist of other elements are also of interest. Systems such as hexagonal lattices that consist of metallic atoms like aluminum with other elements such as silicon express magnetism when cut into armchair nanoribbons ${ }^{50}$. What is interesting about this material is that, as opposed to magnetism that originates from edge states such as in zigzag graphene nanoribbons, these systems have a ferro-magnetism that originates across the width of the nanoribbons as seen in Figure 2c and d. This is useful due to the fact that the edges in nanoribbons are susceptible to reconstructions and edge defects that could alter the magnetic properties of those systems away from what would be desirable. Instead in $\mathrm{AlSi}$, the magnetism is itinerant band magnetism. Furthermore, due to the magnetism not being a result of the edge states, the magnetism is distributed in a more ordered manner over the entire structure rather than decaying towards the center. However this configuration is shown to be stable only up to an $N_{A}$ value of 7 or less. Beyond $N_{A}=7$, the AlSi structures deform strongly. Instead, structures that have silicon atoms occupying the edge states rather than aluminum and silicon, 


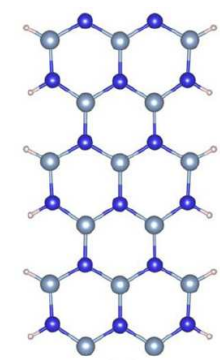

(a)

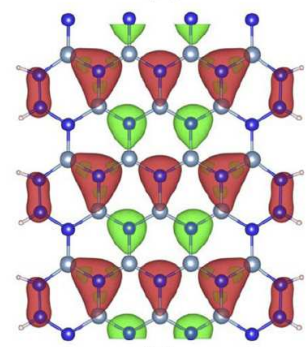

(c)

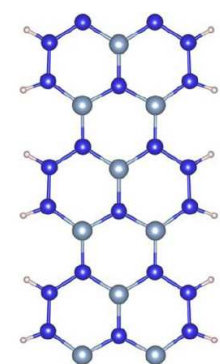

(b)

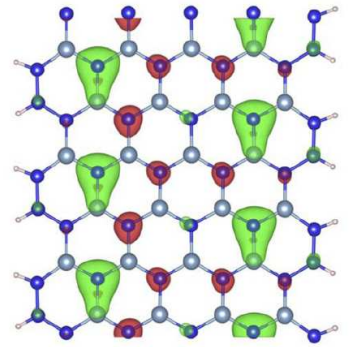

(d)

Fig. 2. (Color online) Structures of AlSi nanoribbons. (a) An equal number of aluminum and silicon atoms along the edges. (b) Only silicon along the edges. (c,d) Spin density of two different sized nanoribbons. Reproduced with permission ${ }^{50}$. Copyright 2012 Elsevier B.V.

as seen in Figure 2b, have a configuration that is more stable and does not exhibit distortion at larger $N_{A}$ values. It is reported though, that the difference in energy between the ferromagnetic and the nonmagnetic states of the AlSi nanoribbons is small, ranging on the order of tens of milli-electron volts. Nonetheless, the results presented here show that magnetism may also be produced in hexagonal systems that is not dependent on the edge states.

Other hexagonal-based one dimensional structures that contain metallic atoms that express magnetism also exist. Specifically when zinc oxide $(\mathrm{ZnO})$, which is ordinarily a nonmagnetic semi-conductor, is doped with non-metal elements, there is a resulting ferromagnetic moment ${ }^{51}$. Carbon, boron, and nitrogen are the three cases of doping presented and their resulting magnetic moments are examined and compared with the nonmagnetic undoped $\mathrm{ZnO}$ structure. It was found that by replacing an oxygen atom with a carbon atom, a ferromagnetic moment arises that is centered on the carbon atom and decays away from the center as seen in Figure $3 \mathrm{a}$ and $\mathrm{b}$. The cause of the magnetic moment is primarily from the strong coupling between the $2 \mathrm{p}$ orbital of carbon and $3 \mathrm{~d}$ orbitals of zinc around the carbon atom. Furthermore, the effect of a second carbon atom dopant was investigated at varying distances between the two carbon atoms. The carbon dopants occupy the lattice positions of two adjacent oxygen atoms and as they are moved further apart, the $\mathrm{ZnO}$ monolayer undergoes a transition from nonmagnetic to anti-ferromagnetic to 

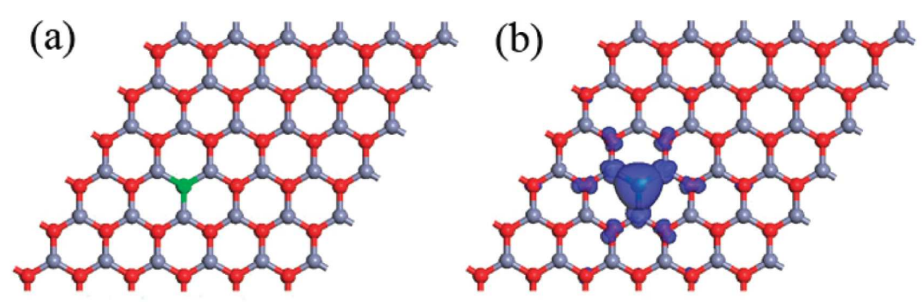

Fig. 3. (Color online) (a) Structure of a $\mathrm{ZnO}$ monolayer with a single carbon dopant atom shown in green. (b) Spin density around the carbon atom dopant. Reproduced with permission ${ }^{51}$. Copyright 2012 American Chemical Society.

ferromagnetic. At their closest distance in which the carbon atoms form a dimer pair, the $\mathrm{ZnO}$ monolayer exhibits a nonmagnetic state. Moving the two atoms apart results in an anti-ferromagnetic moment up to about $6.46 \AA$ until the distance is increased to $8.62 \AA$, in which the $\mathrm{ZnO}$ layer becomes a ferromagnetic semi-conductor. The energy difference between the ferromagnetic and the anti-ferromagnetic states is relatively small, on the order of tens of milli-electron volts and as the distance between the two carbon atoms increases, the energy difference increases as well. However, based on the formation energy required for the system, the most stable state was determined to be the nonmagnetic case in which the carbon atoms were at their closest distance. For the case of the boron doped $\mathrm{ZnO}$ monolayer, the results are similar. With a single boron atom replacing an oxygen atom, there is a net magnetic moment that is centered around the boron atom. Incorporating an additional dopant boron atom results in a nonmagnetic configuration at their closest distance that changes to anti-ferromagnetic and then to ferromagnetic as the distance increases. The configuration with the lowest formation energy is when the two dopant atoms are at their closest distance. In the case of nitrogen doping, the results differ from the carbon and boron systems. Like carbon and boron, a single nitrogen dopant induces a ferromagnetic state, however in the case of two dopant atoms, they do not form a dimer pair. Instead, the two dopant nitrogens maintain their initial positions of the oxygen sites and the system is in an antiferromagnetic state. Furthermore, as the nitrogen atoms are moved further apart into other oxygen lattice sites, the system maintains an anti-ferromagnetic state in which there is a very small difference between the anti-ferromagnetic and the ferromagnetic energies. Similar to the carbon and boron cases however, the system with the lowest formation energy occurs when the two nitrogen dopants are at their closest distance.

\subsection{Mechanical models of graphene}

Much work has been reported on the mechanical modelling of graphene. Although quantum mechanics modeling is accurate, it is limited for small size and 
zero temperature ${ }^{52,53}$. Commonly, models considering finite temperature effects and/or large systems rely on molecular dynamics (MD) modeling. For example, MD simulations were able to reveal anomalous strength characteristics of tilt grain boundaries ${ }^{54}$. Hydrogen functionalization of graphene was also studied by MD simulations in Ref. ${ }^{55}$. First principles simulations and quasi-harmonic approximations were recently used to study temperature dependent elastic constants and ultimate strength of graphene (and graphyne) $)^{56}$.

Alternatives to MD simulations include continuum models ${ }^{57}$, simplified structural models based on beam lattices ${ }^{58}$. Amongst the continuum models, nonlocal elasticity theories have been widely used to account for scale effects at the nanoscale ${ }^{59,59,60,61,62}$.

The validity of simplified (homogenised) continuum approaches was investigated in Ref. ${ }^{63}$ using bond orbital models, showing that for certain deformations, continuum plate theory breaks down when modelling graphene sheets. In the same piece of work, MD simulations permitted to assess the validity of the plate model assumptions when modelling multi-layer graphene structures.

To simulate the rupture of "large" structures or assemblies, an important obstacle remains: the homogenisation of fracturing structures. Homogenisation is indeed limited to the pre-peak behaviour. Conventional approaches based on homogenisation are therefore insufficient to deal with fracture. Promising approaches based on a concurrent coupling of a fine-scale model in the regions where fracture/dislocations occur with homogenised models elsewhere were presented ${ }^{64,65,66,67}$. Alternative methods propose to reduce the computational expense through algebraic model reduction $^{68,69,70,71}$. Ultimately, these approaches aim at permitting to study structures of engineering relevance at an affordable computational cost.

\section{Graphyne and its families}

After the discovery of graphene, many additional two dimensional carbon structures were found to be thermodynamically stable, and have the potential to be used in many new devices due to their unusual properties. Graphyne, graphdi-yne, and graph $n$-yne are all materials of this class. Their mechanical, electrical, and magnetic properties are the subject of continuing investigations, both in their pristine state, and with different functionalizations, dopants, and vacancies in the lattice structure. For each material, the pristine studies will be addressed first, followed by the studies of the modified structures.

Graphyne was first proposed in 1987 by Baughman and Eckardt as part of a larger investigation into the properties of new forms of carbon that had been sporadically reported, but not systematically investigated ${ }^{9}$. Graphyne is of particular interest due to its electronic structure, which is quite different than existing materials, including other carbon based materials such as diamond and graphite. The most common form of graphyne is $\gamma$ graphyne, although several others have been studied as well. If unspecified, $\gamma$ graphyne is assumed. 


\subsection{Graphyne Mechanical Studies}

Several studies using different methodologies have produced a range of values for the Young's Modulus, ultimate stress, and ultimate strain of pristine graphyne. A widely cited molecular dynamics study by Cranford and Buehler ${ }^{72}$ employed two methods to calculate the Young's modulus, a uniaxial constant strain rate approach, and an energy minimization approach.

For the constant strain rate approach, a $100 \AA$ square sheet of graphyne (Fig. 4) was created with non periodic boundary conditions, using hydrogen to stabilize the edges of the sheet, and the ReaxFF potential, which is based on data from $a b$ initio calculations, to describe the inter-atomic potentials. A constant velocity was applied to the atoms, creating a uniform strain rate on the atoms. The edges parallel to the velocity vector are allowed to move in the direction of the velocity, and the other translational degrees of freedom are fixed. The virial stress was calculated for the interior volume of the sheet, neglecting the stress concentration caused by the boundary conditions.

The energy minimization method used the same sheet of graphyne as before, however it applied a finite strain to the system and quasi-statically minimized the potential energy of the system. Young's modulus was obtained by curve fitting the potential energy vs strain curve and solving equation (1).

$$
U=1 / 2 E \epsilon^{2}
$$

The constant strain rate approach yielded the armchair modulus as $532.5 \mathrm{GPa}$ and the zigzag modulus as $700.0 \mathrm{GPa}$, whereas the the energy minimization approach gave $629.4 \mathrm{GPa}$ and $772.0 \mathrm{GPa}$, respectively, which are significantly different. The calculated values for the ultimate stresses are much more consistent. In the armchair and zigzag directions they are approximately $46 \mathrm{GPa}$ and $104 \mathrm{GPa}$, respectively. The differing Young's Modulus values indicate some strain rate dependence, however the similarity of the ultimate stresses indicated it does not effect the breaking strength of the material. It should be noted that the conversion of $\mathrm{N} / \mathrm{m}$ to GPa used a thickness value of $3.20 \AA$, which was determined to be the minimum system potential energy with respect to interlayer distance ${ }^{72}$.

Another study by Zhang et. al. ${ }^{73}$ also used molecular dynamics and the Adaptive Intermolecular Reactive Empirical Bond Order potential (AIREBO) to investigate the properties of 4 different forms of graphyne: $\alpha, \beta, 6,6,12$ (also named delta), and $\gamma$. The $\gamma$ graphyne is formed by using acetylenic linkages to connect the carbon hexagons of graphene ${ }^{72}$. The other forms of graphyne are formed by replacing different percentages of single bonds with acetylenic linkages. $\alpha$ graphyne has $100 \%$, $\beta$ has $66.67 \%, \gamma$ has $33.33 \%$, and $\delta$ has $41.67 \%$ (Fig. 5). These simulations used periodic boundary conditions in the in-plane directions to simulate a small area of an infinitely large sheet of graphyne. In this way, the effects of boundary conditions are avoided. Uniaxial tension was applied using a constant strain rate as before, however fixing the edges parallel to the velocity was not necessary due to the periodic boundary conditions. The stiffness and strength of the different structures were 
(a)

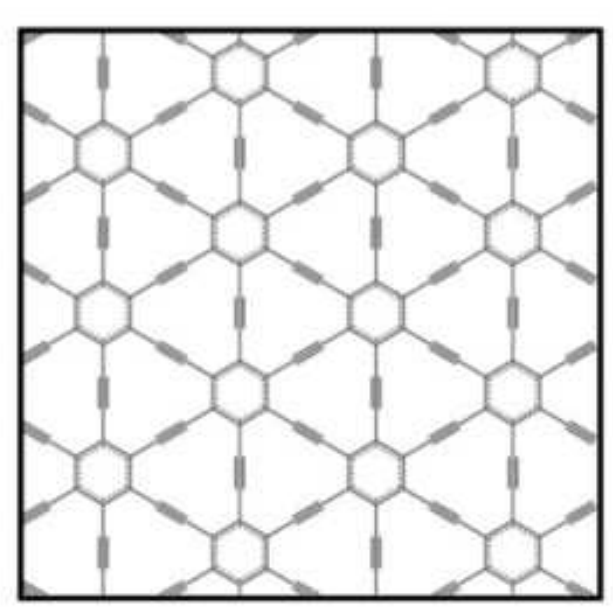

(b)

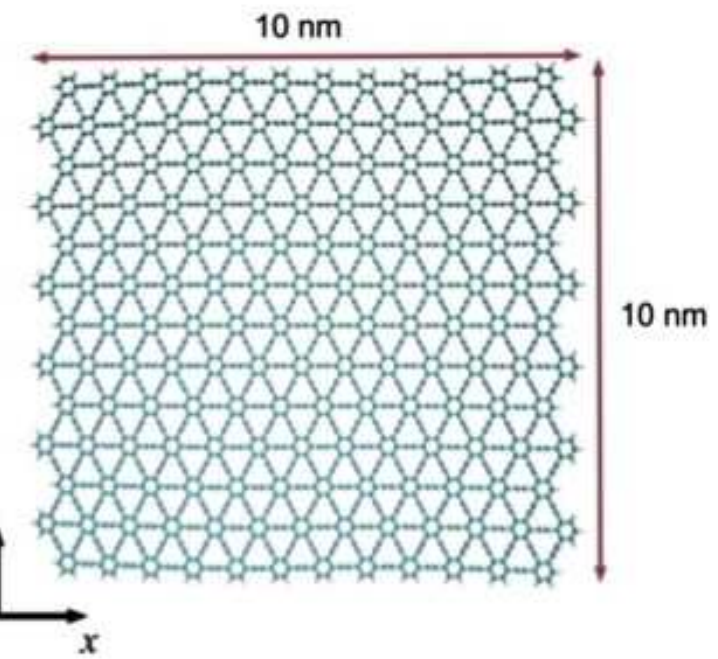

(c)

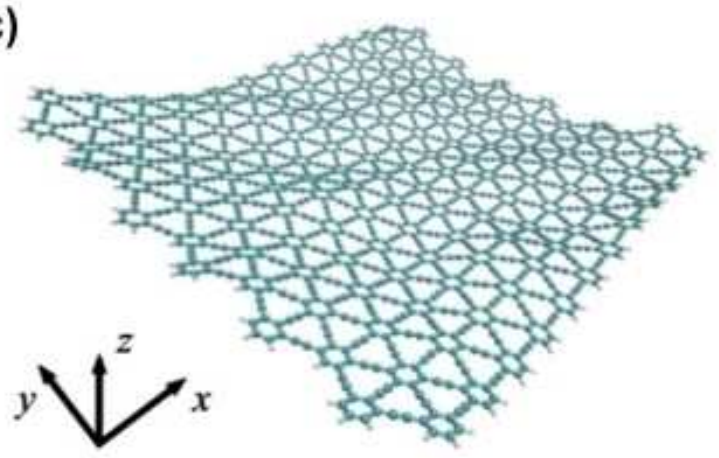

Fig. 4. A: Graphyne chemical structure. B: Graphyne sheet planar view. C: Graphyne sheet 3D view. Reproduced with permission ${ }^{72}$. Copyright 2012 Elsevier. 


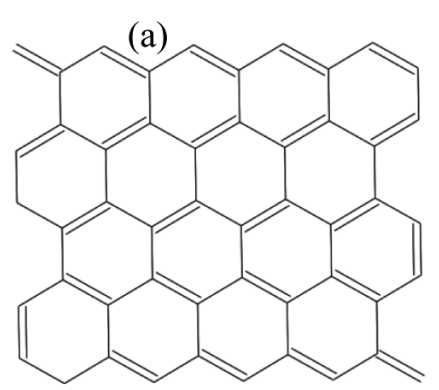

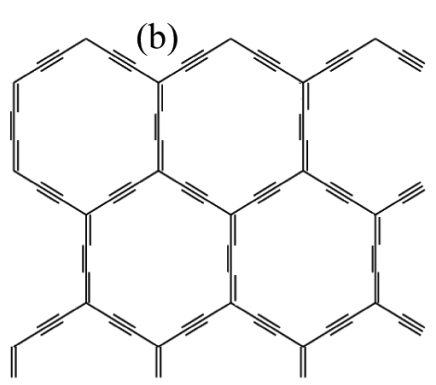
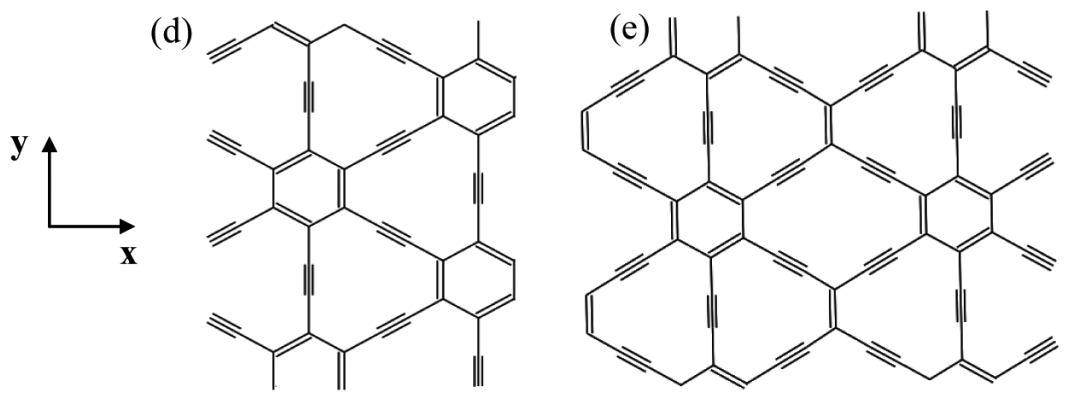

Fig. 5. Chemical structures of A: Graphene. B: $\alpha$ Graphyne. C: $\beta$ Graphyne. D: $\gamma$ Graphyne E: $6,6,12$-Graphyne ( $\delta$ graphyne). Reproduced with permission ${ }^{73}$. Copyright 2013 American Institute of Physics.

found to be inversely proportional to the percentage of acetylenic linkages, making $\gamma$ graphyne the most mechanically favorable structure. Acetylenic linkages form less dense structures by almost a factor of two, resulting in smaller cross sectional areas for the same applied force, causing premature failure.

Comparison of the $\gamma$ form of graphene between the two studies provides interesting results. Zhang et. al. reported an ultimate stress of $63.17 \mathrm{GPa}$ in the armchair direction, and 49.78 in the zigzag direction ${ }^{73}$, compared to 46 and $104 \mathrm{GPa}$, respectively from the first study using ReaxFF. Because the ReaxFF is based on a hybrid potential bond order scheme, using data from first principle quantum mechanics, it is a more accurate description method of the interactions between atoms than AIREBO, although it is more computationally expensive. It is also noteworthy that one study used a finite sized sheet of graphyne called a ribbon, while the other used periodic boundary conditions to create an infinite sheet. The choice of potential functions that balance between accuracy and computational speed is one of the challenges in investigating new materials.

Density Functional Theory (DFT) can also be used to simulate graphyne, and is both more accurate and more computationally expensive than molecular dynamics simulations. Because DFT is based on first principles, rather than an approximation of the potential energy which is used for AIREBO, or the hybrid potential energy-bond order scheme of ReaxFF ${ }^{74}$, it is better suited to investigate systems 
with with large stresses, because it can better model nonlinear behavior. Using DFT, Peng and De ${ }^{75}$ employed a quasi-static method analogous to the energy minimization method employed by Cranford and Buehler to determine the elastic constants of graphyne up to 5 th order. The constants themselves were determined by expressing the components of the Piola-Kirchhoff stress tensor as a Taylor series and curve fitting the stress strain values obtained from DFT calculations ${ }^{75}$. Fourteen independent non zero components were found, and the non-symmetry indicates that graphyne must be anisotropic. Young's modulus was found using the relation between the elastic constants of an anisotropic material and Young's modulus, $Y_{s}=\left(C_{11}-C_{12}\right) / C_{11}$.

The ultimate strengths were also found to be $55.8 \mathrm{GPa}$ for armchair and 58.8 GPa for zigzag, using a thickness of 3.20 A from Cranford and Buehler. The armchair strength is similar to previous studies, however the zigzag strength is not. Zhang et. al. reported a somewhat lower value, while Cranford and Buehler reported a significantly higher value. Graphyne has yet to be fabricated, so experimental data is currently unavailable to clarify these discrepancies.

The studies presented thus far have modeled only pristine graphyne. Several studies have also modeled non pristine graphyne in order to investigate changes to its properties, both mechanical and electro-magnetic. Ajori et. al. ${ }^{76}$ studies two types of vacancies in graphyne, random and mapped, along with pristine graphyne for comparison. A finite sized sheet of $\gamma$ graphyne was used, with approximately $1 \%$ of the atoms in the sheet deleted; for mapped vacancies atom were deleted to form an approximately circular vacancy in the center of the sheet, and for random type vacancies individual atoms at randomly selected locations are deleted. Displacements are applied to the atoms on the ends of the sheet, and the system is relaxed, similar to the quasi-static method of Cranford and Buehler.

The results indicate that the Young's modulus, ultimate stress, and ultimate strain all follow the same trend for both armchair and zigzag. Pristine graphyne has the highest value, followed by mapped defects, followed by random defects (Fig. 6). Poisson's ratio follows the opposite trend ${ }^{76}$. This is expected because a mapped defect removes large numbers of bonds in a small area of the sheet, whereas random defect only remove a few bonds in a small area. As a result, the mapped defect caused the load to be supported by fewer bonds, resulting in failure. Poisson's ratio is a measure of deformation, rather than resistance to deformation, so it is expected to follow the opposite trend of stiffness. It should be noted that the pristine graphyne failed by bond breaking very near the end where the loading was applied. This indicates that the boundary effects may have significantly impacted the ultimate stress and strain values for pristine graphyne. The sheets of graphyne with vacancies did not fail near the boundaries, so the failure behavior is not influenced by the boundary conditions. Because these simulations followed a quasi-static methodology, the failure mechanism may not be accurately modeled because failure is a dynamic phenomenon, and the potential functions used in molecular dynamics do not model large deformations or bond breakage well ${ }^{74}$, and boundary conditions 

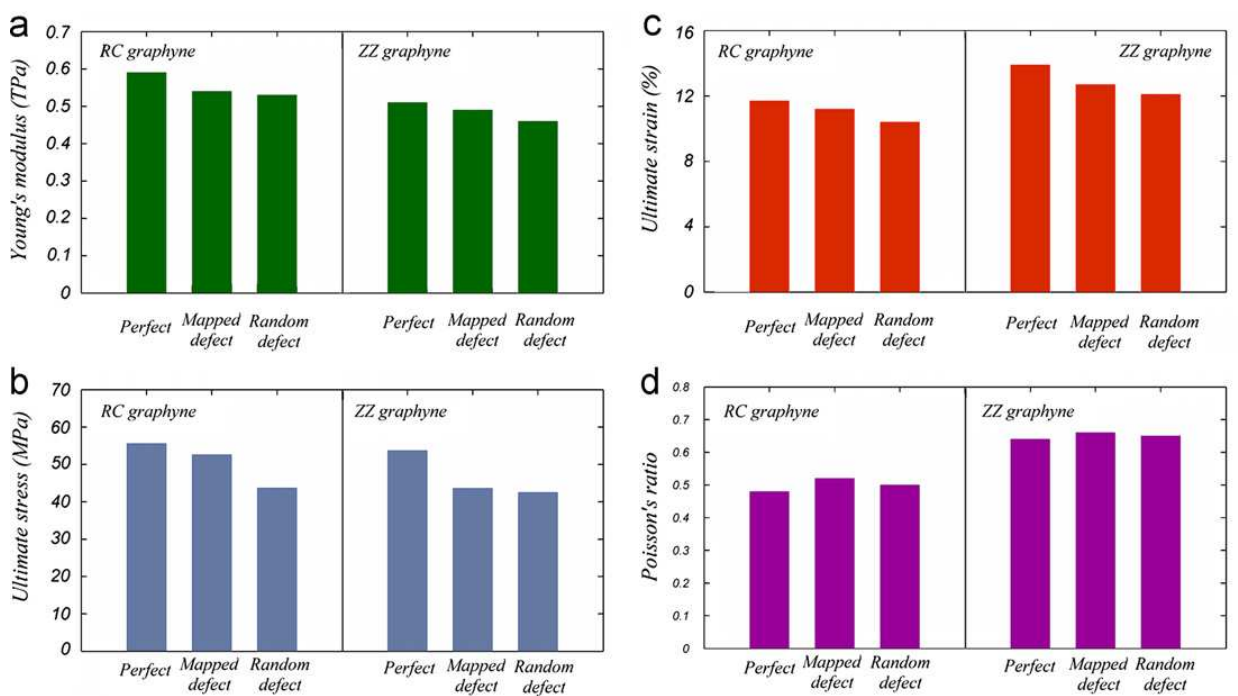

Fig. 6. Results of vacancies in Graphyne. Reproduce with permissions ${ }^{76}$. Copyright 2012 Elsevier.

can introduce stress concentration factors which would influence the results ${ }^{72}$.

Quantitatively, a Young's modulus of $586 \mathrm{GPa}$ was found in the armchair direction, and $510 \mathrm{GPa}$ in the zigzag direction, which is in good agreement with previous results, whereas the ultimate stresses were found to be $55.6 \mathrm{MPa}$ and $53.7 \mathrm{MPa}^{76}$, respectively. The ultimate stress of pristine graphyne was reported as approximately $58 \mathrm{MPa}$. The weakening of graphyne from vacancies is expected, however the ultimate stress is reported as 55.6 MPa, not GPa, despite the correct calculation of Young's modulus. This indicates that some of the results may have been reported in incorrect units. The mechanical properties of graphyne are not significantly reduced by vacancies, indicating a strong potential for the material in design applications.

\subsection{Graphyne Electro-Magnetic Studies}

The electromagnetic properties of graphene and graphyne are of considerable interest, particularly because of the electron transport properties, which may be useful in creating higher performance semi-conductors and transistors than are currently available. Graphyne, both pristine and modified in several ways, has been studied in order to enhance these properties.

The band gap of graphyne nanoribbons of various widths was reported to be in the range of 0.59 to $1.25 \mathrm{eV}$, depending on the width of the nanoribbons, indicating a good semi-conductive material ${ }^{77}$. These corroborate the prediction of Baughman and Eckhardt, who estimated the band gap to $0.79 \mathrm{eV}$, found from the limiting value of a series of similar materials' band gaps ${ }^{9}$.

One study ${ }^{78}$ examined lengthening sides of hexagons of the $\alpha$ graphyne lattice 
with $n$ extra carbon atoms, called $\alpha$ graphyne- $(n)$, using a combination of DFT and $a b$ initio molecular dynamics schemes. It is determined from the phonon frequencies that if $n$ is odd, then the structure is instable, and stable if $n$ is even, although further study at very high numerical accuracy is required due to the sensitivity of phonon frequencies to distant neighbor atoms ${ }^{78}$.

The lengthening of graphyne hexagon edges has significant impact on the electronic properties of graphyne. For the stable structures, Dirac points exist at the Fermi level, and the Fermi velocities are on the order of $10^{5} \mathrm{~m} / \mathrm{s}$, which indicates very high charge carrier mobility, confirming the electrons behave as though nearly massless, confirming the theoretical prediction that electrons appear massless when Dirac points exist near the Fermi level ${ }^{78}$.

The same study also examined the hydrogenation of graphyne, both standard $\alpha$ graphyne and $\alpha$ graphyne- $(n)$. The adsorption of hydrogen widens the band gap of the graphynes, with $\alpha$ graphyne-(2) having a band gap of $5.2 \mathrm{eV}$, becoming an insulator rather than a possible semi-metal or semi-conductor. It is also noted that partial hydrogenation causes a magnetic moment, whereas fully hydrogenated $\alpha$ graphyne-(2) is nonmagnetic ${ }^{78}$. This indicates the possibility of selectively magnetic graphynes, which could be useful in a variety of applications, including sensing. Similar results were obtained for $\gamma$ graphyne and graphdiyne ${ }^{79}$. Graphdiyne will be discussed at length in the next section.

Another approach to enhancing the properties of graphyne is through addition reactions. Oxygen addition by chemical bonding to $\beta$ graphyne has yielded noteworthy results. Graphyne in its pristine state has a zero energy band gap, resulting in transistors with poor performance characteristics ${ }^{80}$, therefore there is significant interest in tuning the band gap. Several configurations of oxygen addition are possible. The most energetically stable is when the oxygen atom lies in the plane of the $\beta$ graphyne sheet, inside the carbon ring made up of 18 atoms. This is particularly important for the electronic structure of the $\beta$ graphyne sheet because the $p_{z}$ orbitals remain uninvolved in the bonding, and the delocalized $\pi$ bonding remains throughout the entire sheet. Different levels of oxygenation are achieved by adding from 1 to 3 oxygen atoms to each ring of 18 carbon atoms. This slightly increases the band gap, $0.2,0.4$, and $0.7 \mathrm{eV}$ for 1,2 and 3 oxygen atoms, respectively ${ }^{80}$. This is particularly important in light of the similar electronic structure of pure and oxygenated graphyne. The delocalized $\pi$ bonding and Fermi velocities on the order of $10^{6} \mathrm{~m} / \mathrm{s}$ remain, while opening the band gap enough to make oxygenated $\beta$ graphyne a useful semi-conductor for transistors. Of particular importance is that increasing oxygenation also increases the band gap.

Rather than chemically reacting graphyne with different atoms, adsorption of transition metals has been simulated in order to investigate changes of the electronic structure of graphyne when empty $3 \mathrm{~d}$ orbitals are introduced ${ }^{81}$. The adoption energy was found to be negative, in the range of -1 to $-4 \mathrm{eV}$, indicating stability of the adsorbed configurations. Adsorption caused a transfer of electrons from the or- 
bitals of the transition metal atom, of which the $3 \mathrm{~d}$ are spin polarized due to the Pauli Exclusion Principle, to the carbon atom. Additionally, electrons within the transition metal change orbitals in a complicated scheme, which tends to reduce the number of unpaired electrons in the transition metal, and increase the number of unpaired electrons in the carbon atom. The s orbitals tend to empty, being excited to a $3 \mathrm{~d}$ orbital, with some $3 \mathrm{~d}$ electrons transferred to the carbon atom. As a result, the carbon atom gains a magnetic moment, and the magnetic moment of the transition metal atom is reduced. The extent of the electron transfer and s orbital promotion is determined by the strength of the attraction of the carbon atom. The strength varies across the transition metals, leading to three distinct groups of transition metal-carbon structures with distinct properties. The first group is half semi-conductors with fully spin polarized electrons, and band gaps of $0.4 \mathrm{eV}$. These are formed from the elements V, Mn, and Co. The second group comes from $\mathrm{Cr}$ and $\mathrm{Fe}$ and show properties of semi-conductors that transition to metals, but remained spin polarized, and the third group is narrow gap semi-conductors, with band gaps of $0.3 \mathrm{eV}$, formed by $\mathrm{Ni}$ adsorption.

Developing tunable band gaps with high charge carrier mobility and selective magnetism properties for graphyne, along with its good mechanical properties, will make graphyne a valuable material for a diverse range of applications, including electronics and sensing.

\subsection{Graphdiyne}

Due to the multiple hybridization states of the carbon atom, many carbon structures with different hybridization schemes are possible. One such structure is graphdiyne (Fig. 7), where the acetylenic linkages of $\gamma$ graphyne are replaced with diacetylenic linkages, effectively doubling the length of the carbon chains connecting the hexagonal rings ${ }^{82}$. It was first predicted by Haley et. al. in $1997^{83}$. Early work was focused on synthesizing the material from similar organic molecules and using computational models of similar materials to estimate the properties of graphdiyne itself ${ }^{83}$. Graphdiyne is part of the graphyne family, however due to its interesting properties, it is typically considered separately ${ }^{84,82}$. Graphdiyne is a softer material than either graphyne or graphene, with an in plane stiffness of $120 \mathrm{~N} / \mathrm{m}$, which is equivalent to a Young's Modulus of $375 \mathrm{GPa}$, if a thickness of $0.320 \mathrm{~nm}$ is assumed ${ }^{82}$. Because of the similarity of graphyne and graphdiyne, this is a reasonable approximation for qualitative comparison. It is also noteworthy that the mechanical behavior of graphdiyne appears to be isotropic ${ }^{84}$.

Because of the superior mechanical properties of graphyne and graphene ${ }^{72,73,86}$, much research has been focused on the electrical and magnetic properties of graphdiyne. As noted above, graphene is a zero band gap material, and the there has been limited success in opening the band gap. Graphdiyne holds promise of a non zero band gap that is also readily tunable.

Using ab initio calculations, $\mathrm{Pei}^{84}$ confirmed the in plane stiffness and calculated 
A

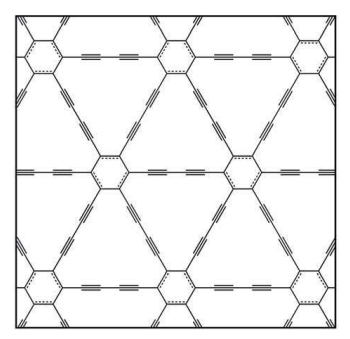

B

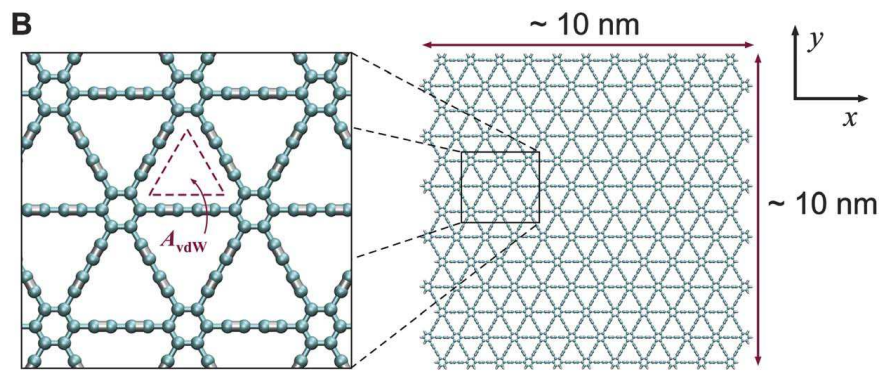

Fig. 7. Structure of Graphdiyne. Reproduced with permissions ${ }^{85}$. Copyright 2012 RSC.

the Poisson's ratio as 0.453 . In a strain free state, the band gap is calculated to be 0.47 and $1.12 \mathrm{eV}$ using two different methods, which clearly indicates the band gap to be non zero. Additionally, the band gap was shown to be proportional (with a positive proportionality constant) to externally applied strain. The band gap varied from $0.28 \mathrm{eV}$ with $\epsilon=-0.05$ to $0.71 \mathrm{eV}$ at $\epsilon=0.06$, with the strain free value of $0.47 \mathrm{eV}$. This shows the band gap of graphdiyne to be readily tunable for specific applications. Additionally, the near massless behavior of electrons, as predicted by the Dirac cone shape of the electron band gap, is verified, resulting in a very attractive semi-conductive material.

Further investigation was conducted by Cui et. al. ${ }^{87}$. Equal biaxial strain was shown to increase the band gap, confirming the previous results, and uniaxial strain was shown to decrease the band gap by changing the electron densities around the carbon atoms in the diacetylenic linkages. The coupling of mechanical loading in electronic properties is very promising for and useful in engineering applications.

Additional methods of electro-mechanical coupling have been explored. In particular, using DFT calculations, the band gap of a graphdiyne nanoribbon, a finite sized sheet of graphdiyne, with some chemical treatment of the edges to stabilize the structure, is shown to be a function of the width of the sheet, with smaller widths associated with larger band gaps ${ }^{77,88}$. The band gap was much more dependent on the width of the zigzag direction than on the armchair direction, indicating some degree of anisotropic behavior of the electronic properties of graphdiyne. One study found the band gap for armchair ranged from 0.59 to $1.25 \mathrm{eV}$, and 0.75 to $1.65 \mathrm{eV}$ for zigzag 77 .

Investigation of graphdiyne's electrical and magnetic properties also includes simulations of electrical and chemical experiments. Some researchers ${ }^{88}$ have modeled the effects of electric fields on the band gap. It was found that an electric field transverse to the ribbon basal plane decreased the band gap, and causes it to become negative for sufficiently large values of the electric field strength. The effect is more pronounced in the armchair direction than in the zigzag direction, and the band gaps with no electric field applied are not equal, confirming some anisotropic behavior of the electronic properties. Also, a semi-conductor-metal transition will 
take place when the band gap becomes small enough, which has significant practical implications, such as in field effect transistors.

As with graphyne, addition of transition metals to graphdiyne changes the electronic properties. The results were discussed in the graphyne section, so they will only be briefly summarized here. The presence of empty $3 \mathrm{~d}$ orbitals affects the degree of delocalized $\pi$ bonding in the carbon lattice, leading to one of three possible outcomes: spin-polarized half-semi-conductors, semi-conductor-metal transition, or narrow gap semi-conductors ${ }^{81}$. The symmetry of the lattice remains intact, so the beneficial electronic properties such as high charge carrier mobility remain. Narrow gap semi-conductors have significant practical value, particularly for thermoelectric devices.

Doping has also been explored as a possible way to enhance the properties of graphdiyne. $\mathrm{Bu}$ et. al. ${ }^{89}$ have investigated the replacement $n$ of pairs of carbon atoms with pairs of boron and nitrogen (BN) atoms. For small doping levels, $n \leq 4$, it is energetically favorable for the $\mathrm{BN}$ pairs to replace the diacetylenic linkage atoms, and for large doping levels, $n \geq 5$, it is energetically favorable for the BN pairs to replace the entire carbon ring. Correspondingly, small doping levels cause a small increase in the band gap, but larger doping levels lead to an approximately linear increase, from $0.53 \mathrm{eV}$ from $n=0$ (pure graphdiyne), to $1.0 \mathrm{eV}$ at $n=4$, to $4.3 \mathrm{eV}$ at $n=10$, which constitutes replacement of all carbon atoms with BN pairs $^{89}$. The change of the two band gap schemes can be explained by the effects of delocalized $\pi$ bonding in the carbon rings, which still occurs to some degree, even when the diacetylenic linkages are replaced with BN. Once the carbon rings are replaced, the band gaps increases significantly.

\subsection{Graphyne Family}

Graphyne and Graphdiyne are two examples of members of the graphyne family. Due to the symmetry of the acetylenic linkage, that is, having a single bond on either end of the linkage, an arbitrary number of acetylenic linkages can be inserted into the carbon chains of the original $\gamma$ graphyne structure, forming graphyne- $n$, where $n$ is the number of acetylenic linkages. In this nomenclature, graphyne-1 is the original graphyne, and graphyne-2 is graphdiyne. Graphynes with higher $n$ values have been investigated to determine their structure and stability, with special interest in their electronic properties. Additionally, structures analogous to graphene and the graphyne family using boron and nitrogen atoms instead of carbon have been predicted, and have generated interest in their electronic and optical properties, and will be discussed in the next section.

The graphyne- $n$ family was examined in detail in a 1998 paper by Narita et. $a l .{ }^{90}$. The structure and electronic properties were investigated for $n=1$ to $n=6$. The lattice constants were determined by finding the configuration with the highest binding energy, indicating stability, as well as the lengths of the bonds. By varying the initial configuration of the system slightly, the possibility that a cumu- 
lative linkage of three double bonds, rather than an acetylenic linkage of one triple bond and two single bonds, would be the most stable configuration for the carbon chains between the hexagons was examined. The most stable configuration was determined to be the acetylenic linkage, results which have been verified for graphyne and graphdiyne in multiple studies ${ }^{72,75,77,88}$. The binding energy is monotonically decreasing with increasing $n$, indicating that large enough $n$ would be unstable. The binding energy for graphyne- 1 is $7.95 \mathrm{eV}$, and $7.66 \mathrm{eV}$ from graphyne- 4 , indicating a slow rate of decrease.

Once the structure had been confirmed, the electronic properties were determined. The effective mass of the electrons was found to be near zero for all configurations studied, and were reported as the ratio of the effective mass to the true mass of an electron, in both the valance and conduction bands. For $n=2$ and $n=4$, the ratio is 0.073 and 0.081 , respectively, in the conduction band and 0.075 and 0.080 in the valance band ${ }^{90}$. The small values indicates the effects of a Dirac point in the energy band gap. The electronic behavior of the odd numbered graphynes is more complex. The effective mass of the electrons exhibits anisotropy depending on which direction the Brillouin zone is traversed. For example, in graphyne-1 traveling from $\Gamma$ to $M$ gives a ratio of 0.15 , whereas traveling from $K$ to $M$ gives a ratio of 0.063 in the conduction band, and similar results in the valence band. The band gaps for all graphynes were found to be in the range of $0.50 \mathrm{eV}$ to $0.60 \mathrm{eV}$, which is consistent with previous studies ${ }^{88,77}$. Due to the similarity of the electronic band structure of graphynes and graphite, Narita et. al. ${ }^{90}$ expects graphynes to respond similarly to alkali metals, opening the possibility of super conduction.

Hexagonal boron nitride (h-BN) has been investigated as a graphene like structure with potential useful properties ${ }^{91}$. Cao et. al. ${ }^{92}$ investigated a graphyne- $n$-like material made from boron and nitrogen $(\mathrm{BN})$, for $1 \leq n \leq 3$. The lattice constants of the BN materials were determined to be slightly larger than the corresponding graphyne- $n$ materials, as were the binding energies, indicating slightly greater energetic stability. The energy gaps were found to be almost an order of magnitude higher, and displaying the opposite trend of energy gap with respect to $n$. The energy gap is found to be $4.202 \mathrm{eV}$ for the BN analogue of graphyne-1, whereas it is $0.473 \mathrm{eV}$ for graphyne- 1 itself, and the BN materials decrease the band gap with increasing $n$, differing from graphynes. From the band structures, the BN analogues of graphyne-1,2, and 3 are wide band gap semi-conductors. The optical properties were also investigated by bombarding the BN structures with radiation, and determining the reflectivity, refractivity index, and energy loss for each. This showed that all structures strongly absorbed ultraviolet (UV) radiation, and that the higher $n$ structures reflected less radiation and had higher energy loss at higher incident photon energies, while having similar indices of refraction ${ }^{92}$.

Significant interest in the properties of graphene and graphyne has lead to many investigations and modifications of these materials, leading to the creation of new variants such as the graphyne-n family, the exploration of BN materials, the discovery of electro-mechanical property coupling, and a number of different function- 
alizations of these materials. The pace of discovery is rapid and accelerating, and the future is sure to bring new discoveries.

\section{Hexagonal Boron Nitride Monolayers}

Hexagonal boron nitride (h-BN) monolayer is an analogue of graphene because it shares a similar structure of 6 atom hexagonal rings. Nitrogen and boron atoms replace the carbon atoms forming an alternating pattern. To form h-BN from two atom lattice shown in Fig. 1, one of the basis atoms is replaced with nitrogen and the other is replaced with boron. Various forms of $\mathrm{BN}$ have existed for some time ${ }^{93}$ and are commercially available. Significant interest in hexagonal BN began a short time ago, spurred by the hope of finding new properties of two dimensional crystal structures, similar to those found in graphene. Mechanical, electrical, and radiation properties have been investigated, and will be addressed in that order.

\section{1. h-BN Mechanical Properties}

The mechanical properties of h-BN are of interest for several reasons such as devising experimental tests of h-BN, acoustics, and the creation of devices from h-BN. Peng et. al. ${ }^{94}$ used Density Functional Theory calculations to determine the elastic constants, up to fifth order, of pure h-BN at zero temperature. Strain was applied to the system and the stress response was measured for armchair, zigzag, and biaxial loading conditions. With no external pressure, $C_{11}=C_{22}$, indicating the h-BN behaves isotropically under ideal conditions, however the material becomes nonisotropic when under external pressure. The in-plane stiffness is reported as 278.3 $\mathrm{N} / \mathrm{m}$. Because the thickness of h-BN is not well known, in-plane stiffness is reported in units of force/distance, rather than Young's modulus in units of force/area. The in-plane stiffness of graphene was calculated as $340 \mathrm{~N} / \mathrm{m}^{76}$, which leads to the conclusion that h-BN is less stiff than graphene. Also, h-BN is shown to have an ultimate stress much lower than graphene.

Peng also investigated the mechanical properties of hybrid h-BN graphene structures $^{95}$ using DFT. Samples were modeled using a repeat unit of 6 ring structures, varying the number of $\mathrm{BN}$ rings from 1 to 6 with all other rings made up of carbon atoms. The in-plane stiffness showed monotonic progression from the pure $\mathrm{h}-\mathrm{BN}$ value to the pure graphene value, and the higher order elastic constants were calculated as before. The elastic constants indicate isotropic behavior, although the strain energy was different in different strain directions ${ }^{96}$. This is expected because in one direction the atomic bonds are parallel to the applied strain and in the other they are at an angle, causing greater force along the bond.

\section{2. $h$-BN Electronic Properties}

The electronic properties of h-BN are of considerable interest for possible semiconductor applications. Unfortunately, many different studies have found conflicting results for the electronic structure of h-BN; the value of the band gap, and 
whether it is direct or indirect have varied widely. These discrepancies were explained by Liu et. al. ${ }^{97}$, who investigated the various possible stacking sequences for h-BN and the effects on the electronic properties. h-BN has been successfully synthesized $^{98,99}$, however isolation of a single layer has been difficult, and the stacking sequence was not well controlled for multi-layer experiments ${ }^{100}$. As a result, the data from several experimental studies has been affected by the stacking sequence. Liu et. al. found three energetically stable stacking sequences, with band gaps of 4.027 (indirect), 3.395 (direct), and 4.208 (indirect) eV. The transient presence of an unstable stacking sequence in a thick structure increases the band gap, which accounts for the variety of experimentally reported values.

The electronic structure of a h-BN monolayer has been investigated using DFT calculations ${ }^{101}$, for both pure h-BN and the h-BN graphene hybrids described in the mechanical properties section. A sheet of pure h-BN is reported to have a band gap of $4.68 \mathrm{eV}$, which agrees well with the results of Liu et. al., although it is slightly higher, which might be due to the effects of other layers on the electronic energy levels. The band gap of the hybrids decreases quadratically with h-BN fraction, to zero band gap for pure graphene ${ }^{80}$. This shows that h-BN can be used to open the band gap of graphene, although charge carrier mobility must still be investigated for the hybrid structures in order to determine if graphene's excellent electron transport properties are affected.

\section{3. h-BN Vacancies and Doping}

The effects of defects, particularly those caused by radiation are of interest in order to design better experimental procedures for investigating h-BN. Kotakoski et. al. ${ }^{102}$ used Transmission Electron Microscopy (TEM) to investigate vacancy formation, discovering that boron atoms are much more likely to be ejected from the lattice than nitrogen atoms under high voltage electron beams. They also used DFT calculation to calculate the energy needed to cause the vacancy formation by atom ejection. They found that $19.36 \mathrm{eV}$ is necessary to eject a boron atom, and 23.06 $\mathrm{eV}$ for a nitrogen atom, confirming the experimental results. Additionally, atoms near the edges of existing vacancies are much more likely to be ejected, requiring 12.92 and $15.00 \mathrm{eV}$ for boron and nitrogen, respectively. The formation energy of such vacancies were found to be a function of applied strain ${ }^{103}$. Both compressive and tensile strain reduced the formation energy of the vacancies. The presence of radiation damage reduced the Young's modulus by 9 percent for boron vacancies and 5 percent for nitrogen vacancies with 0.9 percent of the lattice sites vacant.

The use of TEM to investigate h-BN has the possibility of changing the structure of h-BN or inducing vacancy formation. Okada ${ }^{104}$ investigated the formation of vacancies in h-BN, including conditions similar to those present during TEM, using DFT calculations. For di-vacancies, those with two missing atoms, the removal of one atom of each type, boron and nitrogen, was found to be most favorable under ambient conditions, however when electrons are injected into the system, such as 
what occurs during TEM, 4 atom vacancies, three boron with 1 nitrogen, were more favorable. The exact shape of the vacancy is determined largely by the details of the electron injection. These results explain the formation of vacancies observed by TEM.

A lengthy study by Topsakal et. al. ${ }^{105}$ examined the stability, effects of hydrogen termination, and defects in h-BN. Using DFT calculations, the phonon spectrum was found and indicated stability of the structure in a more rigorous way than the energy methods of Liu et. al. ${ }^{97}$. The band gaps of armchair h-BN nanoribbons with bare edges, armchair hydrogenated edges, and zigzag hydrogenated nanoribbons, indicate they are wide band gap semi-conductors. Depending on the hydrogenation scheme, h-BN may also exhibit ferromagnetic behavior. For zigzag nanoribbons, one edge consists of $\mathrm{N}$ atoms, and the other of $\mathrm{B}$ atoms. If only the $\mathrm{N}$ edge is terminated with hydrogen $(\mathrm{H})$, then the nanoribbon is anti-ferromagnetic and a semi-conductor, compared to a metallic nanoribbon without $\mathrm{H}$ termination. If only the $\mathrm{B}$ edges are terminated, then there nanoribbon exhibits ferromagnetic behavior, which is localized to the $\mathrm{N}$ edge. This is believed to be caused by the interaction of adjacent $\mathrm{N}$ atoms along the unterminated edge, whereas adjacent $\mathrm{B}$ atoms interact in an antiferromagnetic way. Additionally, single vacancies of either boron or nitrogen atoms induce a magnetic moment, whereas a di-vacancy of one boron and one nitrogen is nonmagnetic. The vacancy of a $\mathrm{B}$ atom leads to three unfilled bonds, which gives a total spin of $3 / 2$, causing magnetic moment. $\mathrm{N}$ vacancies cause a magnetic moment $1 / 3$ of $\mathrm{B}$ vacancies.

Vacancies also affect the thermal properties of h-BN. Triangular vacancies, formed by removing all the atoms inside a triangle of a given size, specified by the number of atoms along the base of the triangle, were investigated using nonequilibrium Green's function, in h-BN sheets of finite size ${ }^{106}$. Particular attention was paid to the boundary conditions for the vacancy, and the size of the vacancy relative to the width of the nanoribbon. The thermal transmission coefficient was determined as a function of the phonon frequencies. For a single vacancy in the zigzag direction, the transmission coefficient curve was very similar to that of pure h-BN, although some high frequency phonons are scattered due to the symmetry break at the defect, resulting in slightly lower transmission, and a less smooth curve. For single vacancies with both boron and nitrogen surrounding atoms, the curves are very similar, and show no transmission at a frequency of $422 \mathrm{~cm}^{-1}$, and very small transmission at surrounding frequencies, as though a band stop filter is applied. Yang et. al ${ }^{106}$ explains this using the Density of States to show the energy within the lattice is rotating around the vacancy cite. Further simulations showed that the number of zero transmission frequencies increases with the size of the vacancy. Similar results were obtained for armchair h-BN. As expected from the decreased phonon transmission, the thermal conductance of h-BN decreases with increasing vacancy size. Moreover, the trend is linear. Additionally, wider nanoribbons have larger conductances than narrower ones, which is also expected because the effects of the vacancy are reduced because fewer phonon paths are obstructed 
by the vacancy.

Doping has also been explored in order to understand the causes of the magnetism of defective forms of h-BN. One investigation by Liu and Cheng ${ }^{107}$, doped both $\mathrm{B}$ and $\mathrm{N}$ sites with a wide variety of atom types. If there is an unpaired electron due to the introduction of a dopant atom, a new energy band is formed in between the valence and conduction bands. This leads to a magnetic moment in the doped material. The exchange energy was calculated in order to determine whether the doped materials exhibit long range magnetic order needed for macroscopically observable magnetism. Most of the dopants have small enough exchange energies that they are numerically negligible, however beryllium, carbon, oxygen, silicon, and vanadium, whether doped into a $\mathrm{N}$ or $\mathrm{B}$ site, all show large enough exchange energies to indicate at least paramagnetic behavior on a macroscopic scale.

More research is required for both the electron transport properties of h-BN, theoretically and experimentally, and the mechanical properties, which are important for fabrication and design of devices using this new material. Additional research into functionalizations, including hydrogenation and defect engineering, shows promise of exposing new and useful properties of this two dimensional material.

\section{Silicene and Germanene}

Graphene is an allotrope of carbon, which is a flat monolayer of carbon atoms tightly packed into a two dimensional honeycomb lattice, and is a basic building block for graphitic materials of all other dimensionalities. The Nobel Prize in Physics for 2010 was awarded to Andre Geim and Konstantin Novoselov at the University of Manchester "for groundbreaking experiments regarding the two dimensional material graphene".

Theoretically, graphene has been studied for more than sixty years ${ }^{108}$; it was synthesized on $2004^{1}$ in experiment. After 2004, graphene has been studied extensively both in theory and experiment. Several papers are published every day, and many reviews ${ }^{109,110,111}$ and book chapters ${ }^{112,113}$ have appeared in the last 8 years. These studies span from electronic to optical to excitonic to thermal to mechanical to spin transport to quantum hall effect to functionalization of graphene, etc. Several potential applications for graphene are under development, and many more have been proposed. These include lightweight, thin, flexible, yet durable display screens, electric circuits, and solar cells, as well as various medical, chemical, and industrial processes enhanced or enabled by the use of new graphene materials.

Due to the fascinating properties and extensive applications of graphene, people are perusing and discovering graphene analogues or so-called graphene-like two dimensional materials ${ }^{3}$. A chemical perspective is that one moves down Group 14. The question is that carbon can form a 2-dimensional graphene sheet (in fact, more allotropes); will one find such structures for other members of group 14, Si, Ge, Sn, and $\mathrm{Pb}$ ? 


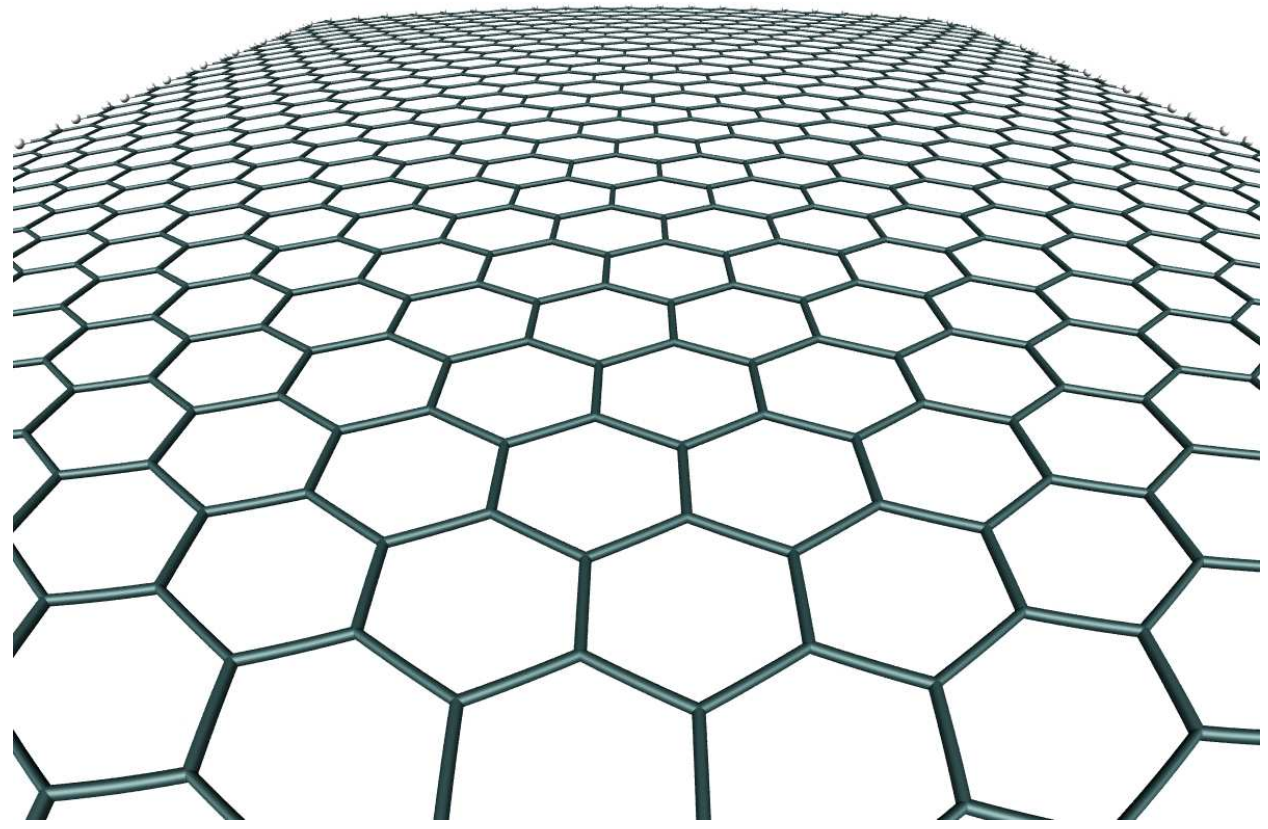

Fig. 8. 2D graphene honeycomb lattice.

To answer the question, in 2010, one of our authors (Wen) theoretically explored group 14 structures across from $1 \mathrm{D}$ to $2 \mathrm{D}$ to $3 \mathrm{D}^{114}$. They suggested that the stability of $\mathrm{C}$ graphene layers, contrasted with the instability of corresponding $\mathrm{Si}, \mathrm{Ge}, \mathrm{Sn}$, and $\mathrm{Pb}$ layers, lead to very different behavior of such layers on aggregation. These graphene layers of $\mathrm{Si}, \mathrm{Ge}, \mathrm{Sn}$, and $\mathrm{Pb}$ have essentially collapsed to $3+2$ or fivecoordinate structures with bonds between layers (see Fig. 9 left). The explanation is that $\pi$-bonding in Group 14, ideal in all the graphene structures, is a good thing only for carbon, and not for $\mathrm{Si}, \mathrm{Ge}, \mathrm{Sn}$, and $\mathrm{Pb}$. In general, greater overlap leads to more stabilization. The same reduced $\pi$ overlap in $\mathrm{Si}=\mathrm{Si}$ double bonds leads to higher reactivity as well; the low-lying $\pi^{*}$, the highlying $\pi$ orbitals are likely to make $\mathrm{Si}=\mathrm{Si}$ highly reactive to bases and acids (see Fig. 9 right). Therefore, the graphene-like sheets of $\mathrm{Si}, \mathrm{Ge}, \mathrm{Sn}$, and $\mathrm{Pb}$ are most unlikely to have an independent existence. If they are to be made, they will have to be intercalated by other atoms or molecules, or substrates or otherwise protected from reacting with each other. In the study, they also predicted that the graphene-type Si structure is semi-metallic, similar to $\mathrm{C}$ graphene, while the $\mathrm{Ge}, \mathrm{Sn}$, and $\mathrm{Pb}$ structures are metallic, with a $\sigma^{*}$ band crossing the Fermi level.

Silicene is the word to name the two dimensional graphene-analogue of silicon. Theorists have predicted the existence and possible properties of silicene ${ }^{114,115,116,117}$. Among these studies, Cahangirov et. al. ${ }^{117}$ predict that silicon 


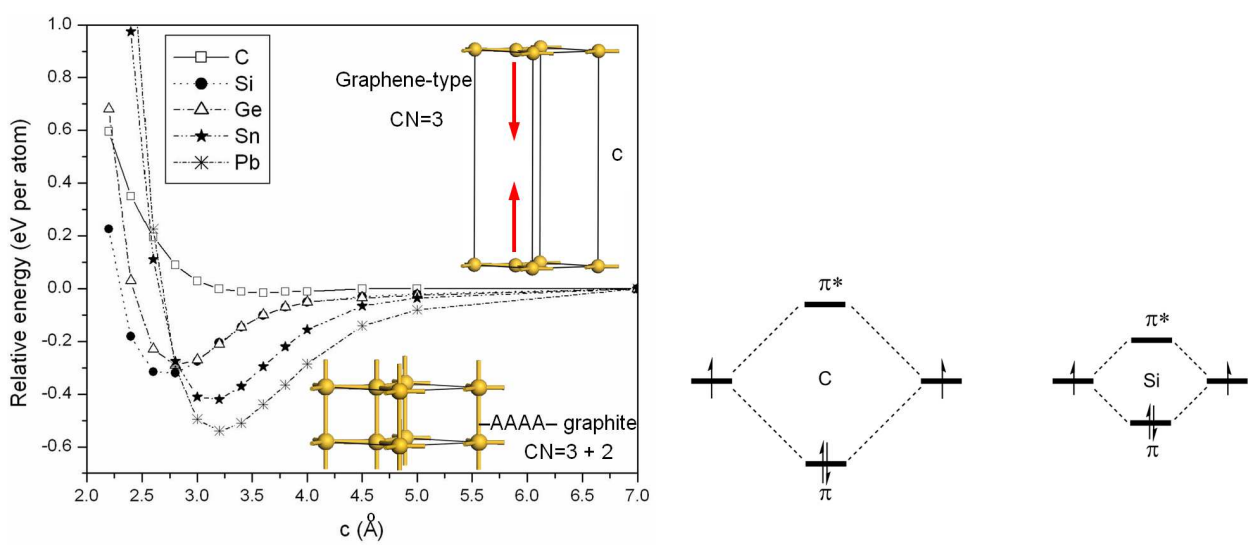

Fig. 9. Left: The relative energy (relative to the corresponding infinitely separated structure) of bringing 2D graphene-type sheets together to form the -AAAA- graphite polytype. Right: A schematic of the p-type overlaps for $\mathrm{C}$ and $\mathrm{Si}$ planar systems. Reprinted with permission from Ref. ${ }^{114}$. Copyright 2010 Wiley-VCH.
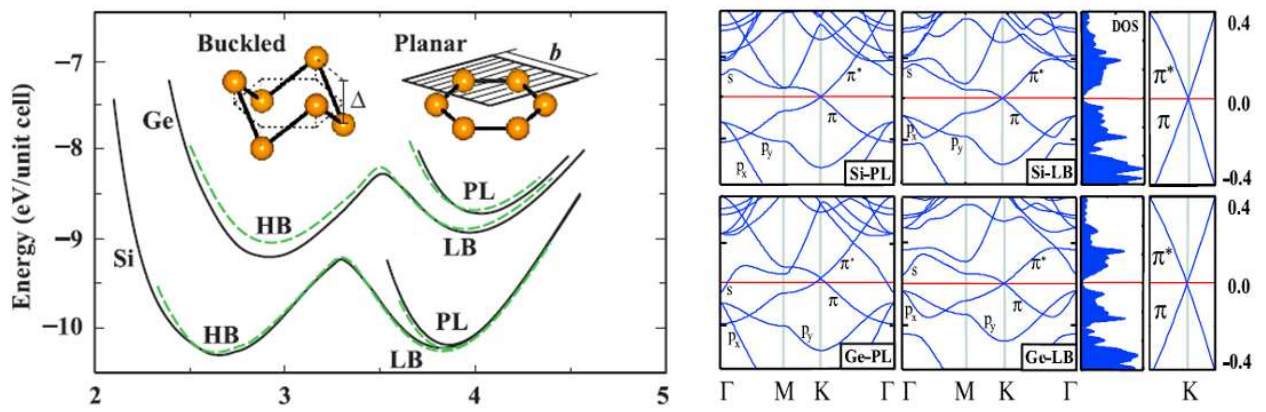

Fig. 10. Energy versus hexagonal lattice constant of $2 \mathrm{D} \mathrm{Si}$ and $\mathrm{Ge}$ are calculated for various honeycomb structures. PL= planar-layer, $\mathrm{LB}=$ low-buckled, and $\mathrm{HB}=$ high-buckled. Reprinted with permission from ref. ${ }^{117}$. Copyright 2009 The American Physical Society.

and germanium can have stable, two dimensional, low-buckled, honeycomb structures which is more stable than their corresponding planar-layer type structures (see Fig. 10 left). Similar to graphene, these puckered structures are ambipolar and their charge carriers can behave like a massless Dirac fermion due to their $\pi$ and $\pi^{*}$ bands which are crossed linearly at the Fermi level (see Fig. 10 right).

The computed phonons of the low-buckled silicene and germanene (a word for graphene-analogue of germanium) by Cahangirov et. al. show they are dynamically stable. However, in their study, they did not point out how to stabilize these 2D structures, as proposed by Wen et. al. ${ }^{114}$.

Though theorists had predicted the existence of silicene, experimentalists first synthesized silicene in $2010^{118,119}$. Using the scanning tunneling microscope (STM), 

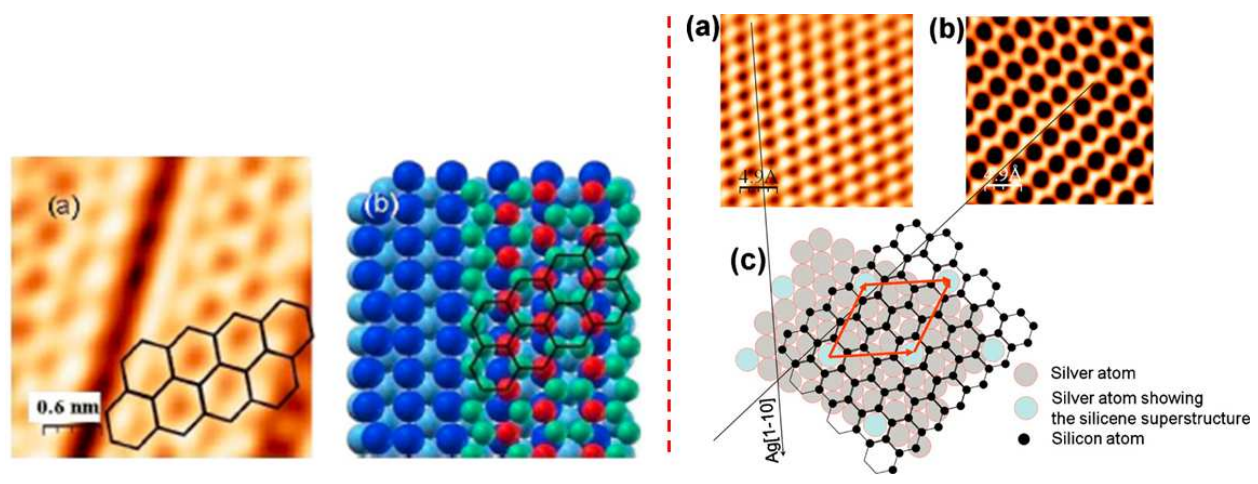

Fig. 11. Left: Silicene on Ag (110); Right silicene on Ag (111). Reprinted with permission from Ref. 118,119 . Copyright 2010 American Institute of Physics.

they studied self-assembled silicene nanoribbons and silicone sheets deposited onto a $\mathrm{Ag}$ surface, $\operatorname{Ag}(110)$ (see Fig. 11 left) and $\operatorname{Ag}(111)$ (see Fig. 11 right), with atomic resolution. The images revealed hexagons in a honeycomb structure similar to that of graphene. Interestingly, unlike silicon surfaces, which are highly reactive to oxygen, the silicene were found to be resistant to oxygen reactivity (as also pointed by Padova et. al. ${ }^{120}$ ). They also performed density functional theory to study the electronic structure and charge density.

In 2012 several groups independently reported ordered phases on the same surface $^{121,122,123,124}$. Especially, Vogt et. al. ${ }^{124}$ provided compelling evidence, from both structural and electronic properties, for the synthesis of epitaxial silicene sheets on a $\mathrm{Ag}$ (111) substrate, through the combination of scanning tunneling microscopy and angular-resolved photo-emission spectroscopy in conjunction with calculations based on density functional theory. They observed that silicene is a buckled type on $\mathrm{Ag}$ (111). Recently, silicene has been reported to grow on a $\mathrm{ZrB}_{2}$ substrate ${ }^{125}$ and $\operatorname{Ir}(111)^{126}$.

Further work on bulk silicene and germanene have been extensively performed $^{127,128,129,130,131}$. These studies cover from electronic properties to the effect of substrates to transport properties to Dirac fermions to mechanical properties to functionalization of silicene to defects and doped silicene.

\subsection{Electronic properties}

As we have talked about the electronic properties above, silicene is semi-metallic, similar to graphene, while the germanene are metallic. Interestingly, theoretical calculations ${ }^{132,133}$ have predicted that applying a transverse electric field across a monolayer buckled silicene and germanene sheets opens a band gap that is proportional to the applied field, and that the structure remains dynamically stable when such an electric field is applied. To put it another way, it is possible to tune the band gap of silicene and germanene via external field, as also suggested by Liang 
et. al. ${ }^{134}$.

\subsection{The effect of substrates}

The p-d hybridization mechanism between $\mathrm{Ag}$ and $\mathrm{Si}$ has been shown to be important to stabilize the nearly flat silicon clusters and the effectiveness of Ag substrate for silicene growth explained by density functional theory calculations and molecular dynamics simulations ${ }^{135}$. Liu et. al. ${ }^{136}$ studied the substrate effect on the electronic properties of silicene, and found that the characteristic Dirac cone is preserved for silicene on h-BN monolayer or hydrogenated Si-terminated $\mathrm{SiC}(0001)$ surface, and the silicene becomes metallic when it is placed on a hydrogenated Cterminated $\mathrm{SiC}(0001)$ surface. Jamgotchian et. al. ${ }^{122}$ experimentally found that the deposition of one silicon monolayer on the silver (111) substrate in the temperature range $150-300{ }^{\circ} \mathrm{C}$ gives rise to a mix of $(4 \times 4),(2 \sqrt{3} \times 2 \sqrt{3}) \mathrm{R} 30^{\circ}$ and $(\sqrt{13} \times \sqrt{13})$ $\mathrm{R} 13.9^{\circ}$ superstructures which strongly depend on the substrate temperature.

\subsection{Dirac states}

The existence of Dirac fermions for silicene on $\mathrm{Ag}(111)$ was observed by scanning tunneling spectroscopy ${ }^{123,137}$. Also, theoretical calculations predicted the existence of Dirac fermions in silicene grown on the $\operatorname{Ag}(111)$ surface by Guo et. al. ${ }^{138}$ who found that Dirac electrons are absent near Fermi energy in all the stable structures due to buckling of the Si monolayer and mixing between $\mathrm{Si}$ and $\mathrm{Ag}$ orbitals.

\subsection{Transport properties}

Density functional theory calculations by Jose $^{139}$ in 2011 suggested that silicene clusters could be excellent materials for FET (field effect transistor) applications and hydrogen storage, and pointed out that stacking of two silicene layers leads to the formation of closed 3D clusters with high symmetry and strong $\mathrm{Si}-\mathrm{Si}$ bonds. Wang et. al. ${ }^{140}$ explored the electron transport through Ag-silicene-Ag junction, and found that before silicene can be grown on or transferred onto insulating substrates, current-in-plane transport measurements do not reflect transport properties of stand-alone silicene. A theoretical calculation by Wang ${ }^{141}$ proposed that the buckled structure, hydrogen saturation, and edge reconstruction as well as edge roughness decrease the carrier mobilities which are explained with the aid of crystal orbitals. The thermal conductivity of silicene studied by $\mathrm{Ng}$ et. al. ${ }^{142}$ suggested that both armchair and zigzag chiralities in silicene have significant differences in thermal conductivities but not in graphene, and graphene possesses significantly higher thermal conductivities than silicene at every length scale and chirality.

\subsection{Mechanical properties}

The mechanical properties of silicene have been studied recently. Studies by Lay et. al. ${ }^{143}$ revealed that pure $\operatorname{Ag}(111)$ surface (a substrate) can mimic a honeycomb 


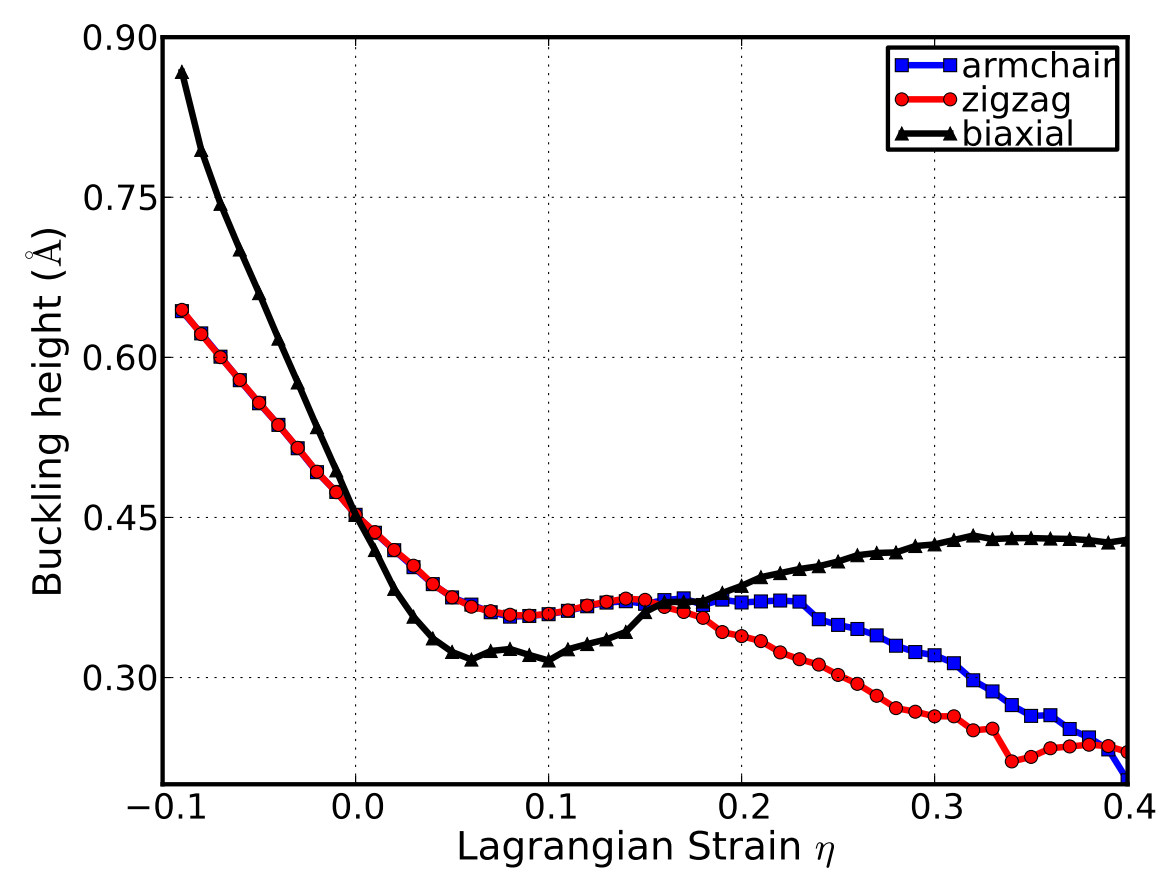

Fig. 12. Both planar and low-buckled silicene are mechanically stable under various strains. Reprinted with permission from Ref. ${ }^{144}$. Copyright 2013 RSC.

structure, which could easily be misinterpreted as a strained silicene layer, and suggested that there is no evidence for the existence of such strong compressively strained silicene layers. In 2013, we have performed DFT studies on the mechanical properties, including the ultimate stresses, ultimate strains, and high order elastic constants, of silicene; our results on the positive ultimate strengths and strains, second order elastic constants, and the in-plane Young's modulus indicate that both planar-type-silicene and low-buckled silicene are mechanically stable (see Fig. 12) ${ }^{144}$.

\subsection{Functionalization of Silicene}

Now, scientists are not only focusing on pure silicene studies, but also perusing more fascinating derivations of silicene, such as hydrogenated silicene (so-called silicane), fluorided silicene, etc. Using DFT, the hydrogenation of silicene was studied by Osborn et. al. ${ }^{145}$ who proposed that partial and patterned hydrogenation, achievable through exposing silicene to hydrogen gas with various densities and/or masking techniques, provide the attractive possibility of metal/semi-conductor/insulator functionality within the same silicon nanosheet. Zhang et. al. ${ }^{146}$ performed first 
principles calculations on the hydrogenation of silicene, and the results show that the weak overlapping between $3 p_{z}$ orbitals of neighbor Si atoms leads to a very reactive surface, resulting in a more energetically stable semi-conducting surface upon being fully hydrogenated. Interestingly, hydrogenation induced magnetic states were found in their study. Besides the external field as discussed above, hydrogenation is another efficient route to tune the electronic properties of silicene sheets. Quhe et. $a l .{ }^{147}$ also pointed out that it is a way to tune the band gap in silicene by surface adsorption. Houssa et. al. ${ }^{148}$ theoretically studied the electronic properties of hydrogenated silicene and germanene, so called silicane and germanane, respectively, and found that these materials are wide band-gap semi-conductors, the type of gap in silicane (direct or indirect) depending on its atomic configuration, while germanane is predicted to be a direct-gap material, independent of its atomic configuration, with an average energy gap of about $3.2 \mathrm{eV}$. Fluorided silicene has been studied in a theoretical way by Ding et. al. ${ }^{149}$ who found that a direct band gap is opened in the silicene fluoride, and the gap values can be continuously modulated by the strain. By breaking the extended $\pi$-bonding network of $\mathrm{Si}$ with $\mathrm{H}$ or $\mathrm{Br}$ atoms on a single side, a team found that the functionalized silicene sheets exhibit long-range FM order with a higher Curie temperature ${ }^{150}$.

\subsection{Defects in Silicene}

Defects not only affect the lattice of a structure, but also cause the changes on mechanical properties, electronic properties, phonons, transport, etc. Recent studies by Zhang et. al. ${ }^{151}$ have shown that such defects can lower phonon thermal conduction in graphene. Li et. al. performed equilibrium molecular dynamic simulations on silicene with and without defects; they found that the in-plane thermal conductivity of silicene sheets is about one order of magnitude lower than that of bulk silicon, and the phonon transport in a silicene sheet is strongly affected by vacancy concentration, vacancy size, and vacancy boundary shape. Using DFT, Song et. $a l .{ }^{152}$ investigated the structural and electronic properties of the armchair silicene nanoribbons with a monovacancy or a di-vacancy. They predicted that either a monovacancy or a parallel oriented di-vacancy changes a direct gap semi-conductor silicene nanoribbons to an indirect one, while a slanting oriented di-vacancy changes it to a metallic character. However, the vacancies do not change the nonmagnetic character of silicene nanoribbons. Furthermore, the optimized vacancy structure and the electronic properties are independent of the vacancy positions relative to the edge of the nanoribbon.

\subsection{Doped Silicene}

In 2011, Cheng et. al. ${ }^{153}$ studied the effects of doping on the lattice structure, electronic structure, phonon spectrum, and electron-phonon coupling of low-buckling silicene. Their results show that although the lattice is found to be very sensitive to the carrier concentration, it is stable in a wide doping range. The studies by Wang 
et. al. ${ }^{154}$ demonstrated that the n-type/zero-band-gap/p-type semi-conducting features can be switched for silicene and germanene by applying strains. Zheng et. $a l .{ }^{155}$ studied the geometric, electronic, and magnetic properties of pristine, $\mathrm{N}$ or $\mathrm{B}$ doped, as well as $\mathrm{N}$ and $\mathrm{B}$ co-doped silicene nanoribbons, and suggested that the substitution of $\mathrm{N}$ or $\mathrm{B}$ for $\mathrm{Si}$ is preferentially at the ribbon edge sites. A singly substituted $\mathrm{N}$ or $\mathrm{B}$ atom at the edges results in a semi-conductor-metal transition in armchair silicene nanoribbons because of the appearance of half-filled impurity band near the Fermi level.

Silicene and germanene have been becoming a hot topic. Kara et. al. ${ }^{156}$ reviewed silicene, a new candidate for electronics by presenting the available experimental and theoretical studies performed to date, and suggest future directions to be explored to make the synthesis of silicene a viable one. There are many unique and fascinating properties of silicene (or germanene) waiting to be predicted and discovered from both experimental and theoretical sides. Let's move on!

\section{Molybdenum disulfide monolayer}

Molybdenum disulfide monolayer belongs to a large family of two-dimensional layered metal chalcogenide materials that have the general formula $\mathrm{MX}_{2}$, where $\mathrm{M}$ is a metal and $\mathrm{X}$ is a chalcogen $(\mathrm{S}, \mathrm{Se}$, or $\mathrm{Te})$. Transition-metal dichalcogenide semi-conductors have attracted much attention in the past, since some of its members exhibited superconducting properties (e.g. $\mathrm{TaS}_{2}{ }^{157}$ and $\mathrm{NbS}_{2}{ }^{158}$ ). Bulk molybdenum disulfide $\left(\mathrm{MoS}_{2}\right)$ is abundant in nature and has been used as solid lubricant due to its very low friction and mechanic robustness. Catalytic effects from $\mathrm{MoS}_{2}$ on hydrodesulfuritation and hydrogen evolution have been known for decades ${ }^{159}$. Bulk $\mathrm{MoS}_{2}$ has been investigated extensively in the past. It is a solid consisting of layers which are bonded through van der Waals forces. Within a layer, each molybdenum atom is covalently bonded to nearby six sulfur atoms, with the bonds mainly consisting of the $4 \mathrm{~d}$ and $3 \mathrm{p}$ orbitals from molybdenum and sulfur (Fig. 13). $\mathrm{MoS}_{2}$ bulk is diamagnetic with an indirect band gap of $1.29 \mathrm{eV}^{160,161}$. Since the discovery of graphene ${ }^{162}$, monolayer $\mathrm{MoS}_{2}$, which is also a two dimensional material, has received enormous attention due to its striking optical, electronic, and mechanic properties ${ }^{10,11}$. Monolayer $\mathrm{MoS}_{2}$ has a direct optical gap of $1.8 \mathrm{eV}^{12,13,14}$. It can be prepared using the scotch tap technique ${ }^{162,163}$, lithiumbased intercalation ${ }^{164,165}$, laser-thinning ${ }^{166}$, solvent exfoliation ${ }^{167,168}$, chemical vapor deposition ${ }^{169,170}$, plasma etching ${ }^{171}$, and liquid-based processing ${ }^{172,173,174}$.

The mechanical properties of monolayer $\mathrm{MoS}_{2}$ have been extensively studied both experimentally and theoretically. Monolayer $\mathrm{MoS}_{2}$ has a stiffness that is comparable to steel with an in-plane stiffness of $180 \pm 60 \mathrm{Nm}^{-1}$, corresponding to an effective Yong's modulus of $270 \pm 100 \mathrm{GPa}^{175}$. It breaks at an effective strain of $6 \sim 11 \%$ with an average breaking strength of $15 \pm 3 \mathrm{GPa}^{175}$. The Young's modulus of free-standing MoS2 nanosheets (with more than two layers) were reported to be $300 \mathrm{GPa}^{176}$, comparable to results from monolayer MoS2. These experiments 

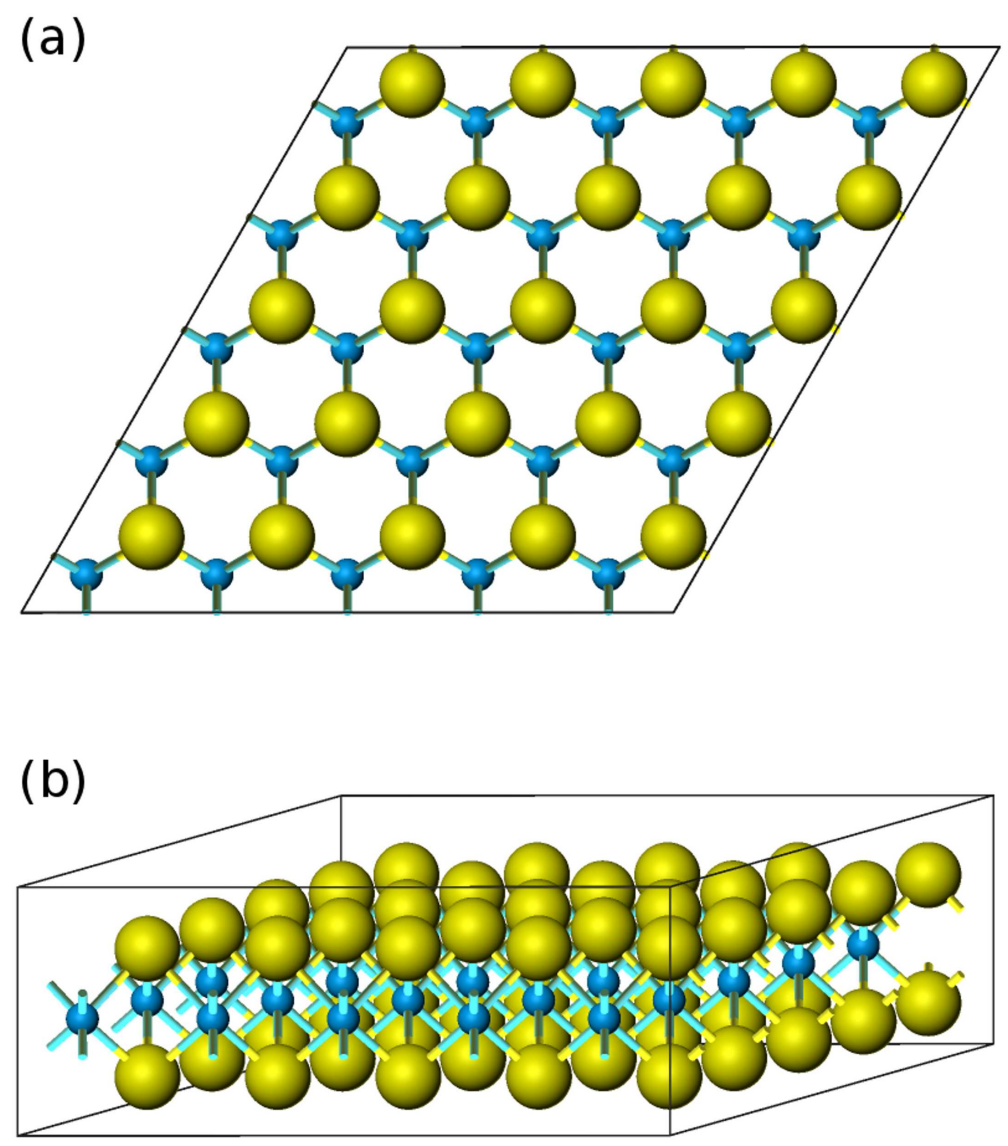

Fig. 13. Structure of monolayer $\mathrm{MoS}_{2}$. (a) Top view: 2-dimensional hexagonal structure (Mo atom: blue, S atom: yellow). (b) Side view: each Mo atom is bonded to six $\mathrm{S}$ atoms

were supported by first-principle simulations ${ }^{177}$. Nanoindentation on monolayer MoS2 was studied with molecular dynamics, Young's modulus was extracted from the simulations and was estimated to $1.0 \mathrm{TPa}$, which is in good agreement with experiment ${ }^{178}$. Nonlinear elastic behavior of MoS2 has also been recently studied both experimentally and from first-principles ${ }^{179}$. Its outstanding mechanical properties and its application in elastic energy storage are reported by very recently ${ }^{180}$.

One appealing property of monolayer $\mathrm{MoS}_{2}$, compared to graphene, is its direct band gap $(\sim 1.8 \mathrm{eV})$, which makes it a promising candidate for building nano-scale electronics. However its mobility is $0.5 \sim 3 \mathrm{~cm}^{2} / \mathrm{Vs}$, which is far below the mobility of graphene, which is $2000 \sim 5000 \mathrm{~cm}^{2} / \mathrm{Vs}^{162}$. Such a low mobility undoubt- 
edly prevents MoS2 from being used as building blocks for nano-scale electronics, such as field-effect transistors (FET). However, a recent experiment ${ }^{15}$ showed that by employing materials with high dielectric constant for the gate, the mobility of monolayer MoS2 can be greatly enhanced to at least $200 \mathrm{~cm}^{2} / \mathrm{Vs}$, with an excellent current on/off ratio of $10^{8}$ at room temperature ${ }^{15}$. Such enhancement is mainly due to the dielectric screening from the gate material as predicted by theoretical calculations ${ }^{181}$. Such findings put $\mathrm{MoS}_{2}$ back on track to become a very promising candidate for the channel materials in the interband tunnel $\mathrm{FET}^{16}$ and transparent transistors used in solar cells. To rationalize these exciting discoveries, first-principle quantum transport simulations ${ }^{182}$ have been carried out to study how well the transistor based on monolayer $\mathrm{MoS}_{2}$ could perform. From simulations, an effective mass of $m^{\star}=0.45 m_{0}$ was found which indicates that monolayer $\mathrm{MoS}_{2}$ could be used for slow-speed and low-power transistors. The breakdown current density on monolayer MoS2 transistor was recently reported to be $5 \times 10^{7} \mathrm{~A} / \mathrm{cm}^{2}$, which is even 50 times larger than the breakdown current in copper ${ }^{183}$. It is also experimentally demonstrated that a monolayer MoS2 transistor can be fabricated on ion gel film ${ }^{184}$. In that study, a reasonable mobility of $12.5 \mathrm{~cm}^{2} / \mathrm{Vs}$ and a on/off current ratio of $10^{5}$ was obtained, which makes it promising to build flexible electronics based on MoS2.

The magnetic and electronic properties of MoS2 nanoribbons with either zigzagor armchair-terminated edges were theoretically studied ${ }^{185}$. The zigzag nanoribbons were found to be metallic and ferromagnetic, irrespective of the ribbon width and thickness. Armchair nanoribbons were found to be nonmagnetic and have direct band gaps, with a band gap gradually converging to $0.56 \mathrm{eV}$ with an increasing ribbon width. Armchair monolayer MoS2 was reported to be mechanically stable ${ }^{186}$.

Another exciting application of MoS2 is "valleytronics", which was originally proposed for graphene ${ }^{20,21,22}$. Valleytronics exploits the fact that electrons with equal energy and sitting at different "valleys" in the conduction and valence bands possess different momentum. By modulating electron numbers in valleys, valleytronics would be realized. Photo-induced spin Hall and valley Hall effects that generates long lived spin and valley accumulation at sample boundaries were also proposed $^{19}$. Very recently, the control of electron population in valleys was realized for MoS2 $2^{17,18,19}$ through optical pumping. A "valley polarization" of $30 \%$ has been reported ${ }^{17}$, and can be maintained for more than one nanosecond. Valleytronics based on MoS2 would make it possible to transport charge and spin at the same time.

Besides applications in FET and valleytronics, phototransistors based on monolayer MoS2 were also recently demonstrated ${ }^{23}$. The switch speed is very appealing: the photocurrent can be completely switched between on and off within $50 \mathrm{~ms}$. The photoresponsivity can reach $7.5 \mathrm{~mA} / \mathrm{W}$ with an incident light of a power about 80 $\mu \mathrm{W}$ and a gate voltage of $50 \mathrm{~V}$. In Ref. ${ }^{24}$, it also demonstrated that the absorption spectrum can be controlled by changing the thickness of MoS2 from monolayer to multi-layers. Gas sensors based on MoS2 was also experimentally realized ${ }^{25}$ for detecting nitric oxide. In that study, monolayer MoS2 gave an unstable response 
current, however two-, three-, and four-layer MoS2 sensors yielded stable currents and reached a detection sensitivity of $0.8 \mathrm{ppm}$. Other applications based on MoS2 include catalytic hydrodesulfonization, hydrogen evolution ${ }^{28,29}$, photoelectrochemcial hydrogen production ${ }^{30,31,32,33}$, small-signal amplifier ${ }^{34}$, and solid lubricant ${ }^{35}$. To better understand the catalytic effect of MoS2, the dependence of morphological and electronic structure on the number of layers and sizes were studied extensively in the past ${ }^{26,187,188}$.

\section{CONCLUSIONS}

In summary, we reviewed the defect engineering the properties of the $2 \mathrm{D}$ materials with monatomic thick layers. We examined the effects of defects and dopants in modifying the magnetic properties of graphene and graphene-like structures. It was shown that through the use of vacancies that introduce different edge states, magnetism may be induced within the structures. Edges such as zigzag states are able to induce ferrimagnetism in a local area within the structures that decays as the distance increases away from the states. In addition to vacancies, dopants and hybrid structures may also result in induced magnetism.

Studies of graphyne and its family of materials have uncovered some useful properties. The different bonding structures of graphyne- 1 and the changes in electronic properties of the $n$ graphynes give a rich set of properties even in the pristine state, and the introduction of vacancies, adsorbed atoms, external electric fields, and mechanical strains provide ample means to modify these properties, most importantly the band gap. Graphyne has not yet been synthesized, although research is continuing, and will be needed to provide experimental data against which to test the computational predictions as well as to clarify some discrepancies regarding mechanical properties.

Hexagonal boron nitride is an analogue of graphene which shows some impressive properties including stiffness and strength, and has useful electronic properties as well, such as wide band gap semi-conduction. The ability to combine graphene and h-BN to form a hybrid structure shows promise for future property modulation and control. The effects of vacancies have been studies by both computational and experimental means, and h-BN shows show magnetic behavior for some types of vacancies and for substitutional doping of certain dopants. Multi-layer h-BN has been fabricated and used for some experimental studies, although more work is needed to isolate a single layer. h-BN holds promise of being a useful material for both mechanical and electronic applications.

Silicene and germanene, the graphene equivalents for silicon and germanium, could follow this trend, opening new perspectives for applications, especially due to their compatibility with Si based electronics. Silicene and germanene would share essentially the same electronic properties as graphene, namely an electronic dispersion resembling that of relativistic Dirac fermions at the $\mathrm{K}$ points of the Brillouin-zone, the so-called Dirac points. However, isolated silicene does not yet 
exist in our world. In experiment, silicene was reported to grow on certain surfaces/substrates which play an important role in stabilizing silicene. In fact, these substrates might influence the application of silicene. In addition, the effect of defects in silicene/germanene on the electronic transport and mechanical properties are still scarcely known. It is true that further experimental and theoretical investigations are needed.

It has only been nine years since the "discovery" of graphene. The graphene-like $2 \mathrm{D}$ systems are still in their infancy. However, the known outstanding properties of such materials provide excellent motivation for expanding research activities in this rapidly emerging field. Using advanced nanotechnologies such as accurate atom manipulation, defect engineering that leads to the discovery of exotic properties makes this field of $2 \mathrm{D}$ crystals a fertile one for future investigation and emerging technological applications with precisely tailored properties.

\section{ACKNOWLEDGEMENTS}

The authors would like to acknowledge the generous financial support from the Defense Threat Reduction Agency (DTRA) Grant \# BRBAA08-C-2-0130 and \# HDTRA1-13-1-0025.

\section{References}

1. K. S. Novoselov, A. K. Geim, S. V. Morozov, D. Jiang, Y. Zhang, S. V. Dubonos, I. V. Grigorieva, and A. A. Firsov. Electric field effect in atomically thin carbon films. Science, 306(5696):666-669, 2004.

2. K. S. Novoselov, D. Jiang, F. Schedin, T. J. Booth, V. V. Khotkevich, S. V. Morozov, and A. K. Geim. Two-dimensional atomic crystals. Proc. Nat. Acad. Sci., USA, 102(30):10451-10453, JUL 262005.

3. Mingsheng $\mathrm{Xu}$, Tao Liang, Minmin Shi, and Hongzheng Chen. Graphene-like twodimensional materials. Chem. Rev., 113(5):3766-3798, 2013.

4. Qing Peng, Chao Liang, Wei Ji, and Suvranu De. A first principles investigation of the mechanical properties of g-TIN. Model. Numer. Simul. Mater. Sci., 2:76-84, 2012.

5. Qing Peng, Chao Liang, Wei Ji, and Suvranu De. A first principles investigation of the mechanical properties of $\mathrm{g}-\mathrm{ZnO}$ : the graphene-like hexagonal zinc oxide monolayer. Comput. Mater. Sci., 68:320-324, 2013.

6. Qing Peng, Chao Liang, Wei Ji, and Suvranu De. A first-principles study of the mechanical properties of g-GeC. Mech. Mater., 64:135-141, 2013.

7. Qing Peng, Xiao-Jia Chen, Sheng Liu, and Suvranu De. Mechanical stabilities and properties of graphene-like aluminum nitride predicted from first-principles calculations. $R S C$ Adv., 3:7083-7092, 2013.

8. Qing Peng, Zhongfang Chen, and Suvranu De. A density functional theory study of the mechanical properties of graphane with van der waals corrections. Mech. Adv. Mater. and Struc., 2013.

9. R. H. Baughman, H. Eckhardt, and M. Kertesz. Structure-property predictions for new planar forms of carbon: Layered phases containing $\mathrm{sp}^{2}$ and $\mathrm{sp}$ atoms. J. Chem. Phys., 87:6687-6699, dec 1987. 
10. M. Chhowalla, H. S. Shin, G. Eda, L.-J. Li, K. P. Loh, and H. Zhang. Nature Chemistry, 5:263, 2013.

11. X. Huang, Z. Zeng, and Hua Zhang. Chem. Soc. Rev., 42:1934, 2013.

12. K. F. Mak, C. Lee, J. Hone, J. Shan, and T.F. Heinz. Phys. Rev. Lett., 105:136805, 2010.

13. S. Lebègue and O. Eriksson. Electronic structure of two-dimensional crystals from ab initio theory. Phys. Rev. B, 79(11):115409, March 2009.

14. A. Kuc, N. Zibouche, and T. Heine. Influence of quantum confinement on the electronic structure of the transition metal sulfide $\mathrm{TS}_{2}$. Phys. Rev. B, 83:245213, June 2011.

15. B. Radisavljevic, A. Radenovic, J. Brivio, V. Giacometti, and A. Kis. Nature Nanotech., 6:147, 2011.

16. S. Banerjee, W. Richardson, J. Coleman, and A. Chatterjee. Electron Dev. Lett., 8:347, 1987.

17. H. Zeng, J. Dai, W. Yao, D. Xiao, and X. Cui. Valley polarization in $\mathrm{MoS}_{2}$ monolayers by optical pumping. Nature Nanotech., 7:490-493, 2012.

18. K. F. Mak, K. He, J. Shan, and T.F. Heinz. Nature Nanotech, 7:494, 2012.

19. D. Xiao, G.-B. Liu, W.X. Feng, X.D. Xu, and W. Yao. Phys. Rev. Lett., 108:196802, 2012.

20. A. Rycerz, J. Tworzydlo, and C. W. J. Beenakker. Nature Physics, 3:172, 2007.

21. D. Xiao, W. Yao, and Q. Niu. Phys. Rev. Lett., 99:236809, 2007.

22. W. Yao, D. Xiao, and Q. Niu. Phys. Rev. B, 77:235406, 2008.

23. Z.Y. Yin, H. Li, H. Li, L. Jiang, Y. Shi, Y.H. Sun, G. Lu, Q. Zhang, X.D. Chen, and H. Zhang. Single-layer MoS2 on mica: studies by means of scanning force microscopy. ACS Nano, 6:74, 2012.

24. H.S. Lee, S.-W. Min, Y.-G. Chang, M. K. Park, T. Nam, H. Kim, J. H. Kim, S. Ryu, and S. Im. Nano Lett., 12:3695, 2012.

25. H. Li, Z.Y. Yin, Q.Y. He, H. Li, X. Huang, G. Lu, D.W.H. Fam, A.I.Y. Tok, Q. Zhang, and H. Zhang. Small, 8:63, 2012.

26. T.S. Li and G. L. Galli. J. Phys. Chem. C, 111:192, 2007.

27. Xiao-Dong Wen, Tao Zeng, Yong-Wang Li, Jianguo Wang, and Haijun Jiao. Surface structure and stability of mosx model clusters. The Journal of Physical Chemistry B, 109(39):18491-18499, 2005.

28. B. Hinnemann, P.G. Moses, J. Bonde, K.P. Jorgensen, J.H. Nielsen, S. Horch, I. Chorkendorff, and J.K. Norskov. J. Am. Chem. Soc., 127:5308-5309, 2005.

29. T. F. Jaramillo, K. P. Jrgensen, J. Bonde, J. H. Nielsen, S. Horch, and I. Chorkendorff. Science, 317:100, 2007.

30. B. K. Miremadi and S. R. Morrison. J. Catal., 103:334, 1987.

31. C. T. Tye and K. J. Smith. Catal. Today, 116:461, 2006.

32. E. Fortin and W. M. Sears. J. Phys. Chem. Solids, 43:881, 1982.

33. F. Cesano, S. Bertarione, A. Piovano, G. Agostini, M. M. Rahman, E. Groppo, F. Bonino, D. Scarano, C. Lamberti, S. Bordiga, L. Montanari, L. Bonoldi, R. Millini, and A. Zecchina. Catal. Sci. Technol., 1:123, 2011.

34. M. B. Whitwick B. Radisavljevic and A. Kis. Appl. Phys. Lett., 101:043103, 2012.

35. M. Chhowalla and G. A. J. Amaratunga. Thin films of fullerene-like mos 2 nanoparticles with ultra-low friction and wear. Nature, 407:164-167, September 2000.

36. Simone Bertolazzi, Daria Krasnozhon, and Andras Kis. Nonvolatile memory cells based on mos2/graphene heterostructures. ACS Nano, 7(4):3246-3252, 2013.

37. ZJ Wang and XM Qiu. Two theorems on the Hubbard-Hirsch model. Commun. Theor. Phys., 28(1):51-56, JUL 301997. 
38. S Fajtlowicz, PE John, and H Sachs. On maximum matchings and eigenvalues of benzenoid graphs. Croat. Chem. Acta, 78(2):195-201, JUN 2005. Bioinformatics Session of the MATH/CHEM/COMP Meeting 2004, Dubrovnik, CROATIA, JUN 21-26, 2004.

39. Hamed Karimi and Ian Affleck. Towards a rigorous proof of magnetism on the edges of graphene nanoribbons. Phys. Rev. B, 86(11), SEP 262012.

40. Somnath Bhowmick, Amal Medhi, and Vijay B. Shenoy. Sensory-organ-like response determines the magnetism of zigzag-edged honeycomb nanoribbons. Phys. Rev. B, 87(8), FEB 72013.

41. S. Perumal, B. Minaev, and H. Agren. Spin-spin and spin-orbit interactions in nanographene fragments: A quantum chemistry approach. J. Chem. Phys., 136(10), MAR 142012.

42. M. Grujic, M. Tadic, and F. M. Peeters. Antiferromagnetism in hexagonal graphene structures: Rings versus dots. Phys. Rev. B, 87(8), FEB 202013.

43. Sankaran Lakshmi, Stephan Roche, and Gianaurelio Cuniberti. Spin-valve effect in zigzag graphene nanoribbons by defect engineering. Phys. Rev. B, 80(19), NOV 2009.

44. Ricardo Faccio and Alvaro W. Mombru. Magnetism in multivacancy graphene systems. J. Phys.-Condes. Matter, 24(37), SEP 192012.

45. Min Kan, Jian Zhou, Qiang Sun, Qian Wang, Yoshiyuki Kawazoe, and Puru Jena. Tuning magnetic properties of graphene nanoribbons with topological line defects: From antiferromagnetic to ferromagnetic. Phys. Rev. B, 85(15), APR 252012.

46. Bin Wang and Sokrates T. Pantelides. Magnetic moment of a single vacancy in graphene and semiconducting nanoribbons. Phys. Rev. B, 86(16), OCT 232012.

47. Guoqing Ning, Chenggen $\mathrm{Xu}$, Ling Hao, Olga Kazakova, Zhuangjun Fan, Hao Wang, Kunxun Wang, Jinsen Gao, Weizhong Qian, and Fei Wei. Ferromagnetism in nanomesh graphene. Carbon, 51:390-396, JAN 2013.

48. Liang Feng Huang, Guo Ren Zhang, Xiao Hong Zheng, Peng Lai Gong, Teng Fei Cao, and Zhi Zeng. Understanding and tuning the quantum-confinement effect and edge magnetism in zigzag graphene nanoribbon. J. Phys.-Condes. Matter, 25(5), FEB 6 2013.

49. Yungang Zhou, Zhiguo Wang, Ping Yang, and Fei Gao. Novel Electronic and Magnetic Properties of Graphene Nanoflakes in a Boron Nitride Layer. J. Phys. Chem. $C, 116(13): 7581-7586$, APR 52012.

50. Xi Chen and Jun Ni. Predicted ferromagnetism in hole doped armchair nanoribbons: A first principles study. Chem. Phys. Lett., 555:173-177, JAN 32013.

51. Hongyan Guo, Yu Zhao, Ning Lu, Erjun Kan, Xiao Cheng Zeng, Xiaojun Wu, and Jinlong Yang. Tunable Magnetism in a Nonmetal-Substituted ZnO Monolayer: A First-Principles Study. J. Phys. Chem. C, 116(20):11336-11342, MAY 242012.

52. Qing Peng, Chao Liang, Wei Ji, and Suvranu De. A theoretical analysis of the effect of the hydrogenation of graphene to graphane on its mechanical properties. Phys. Chem. Chem. Phys., 15:2003-2011, 2013.

53. Qing Peng, Chao Liang, Wei Ji, and Suvranu De. Mechanical properties of g-GaN: A first principles study. Appl. Phys. A, pages DOI:10.1007/s00339-013-7551-4, 2013.

54. Rassin Grantab, Vivek Shenoy, and Rodney Ruoff. Anomalous strength characteristics of tilt grain boundaries in graphene. Science, 330(6006):946-948, 2010.

55. Q.X. Pei, Y.W. Zhang, and V.B. Shenoy. A molecular dynamics study of the mechanical properties of hydrogen functionalized graphene. Carbon, 48, 2010.

56. Tianjiao Shao, Bin Wen, Roderick Melnik, Shan Yao, Yoshiyuki Kawazoe, and Yongjun Tian. Temperature dependent elastic constants and ultimate strength of graphene and graphyne. J. Chem. Phys., 137(19), 2012. 
57. C. D. Reddy, S. Rajendran, and K. M. Liew. Equivalent continuum modeling of graphene sheets. Int. J. Nanosci., 04, 2005.

58. F. Scarpa, S. Adhikari, and R. Chowdhury. The transverse elasticity of bilayer graphene. Phys. Lett. A, 374, 2010.

59. S.C. Pradhan and J.K. Phadikar. Small scale effect on vibration of embedded multilayered graphene sheets based on nonlocal continuum models. Phys. Lett. A, 373, 2009.

60. T. Murmu and S.C. Pradhan. Small-scale effect on the free in-plane vibration of nanoplates by nonlocal continuum model. Physica E, 41, 2009.

61. S Pradhan and J Phadikar. Scale effect and buckling analysis of multilayered graphene sheets based on nonlocal continuum mechanics. J. Comput. Theoret. Nanoscience, 7(10):1948-1954, 2010.

62. Le Shen, Hui-Shen Shen, and Chen-Li Zhang. Nonlocal plate model for nonlinear vibration of single layer graphene sheets in thermal environments. Comput. Mater. Sci., 48, 2010.

63. D-B Zhang, E Akatyeva, and T Dumitric. Bending ultrathin graphene at the margins of continuum mechanics. Phys. Rev. Lett., 106(25), 2011.

64. S.P. Xiao and T. Belytschko. A bridging domain method for coupling continua with molecular dynamics. Comput. Methods Appl. Mech. Eng., 193, 2004.

65. Mei Xu, Robert Gracie, and Ted Belytschko. A continuum-to-atomistic bridging domain method for composite lattices. Int. J. Numer. Methods Eng., 81(13):16351658, 2010.

66. Robert Gracie and Ted Belytschko. An adaptive concurrent multiscale method for the dynamic simulation of dislocations. Int. J. Numer. Methods Eng., 86(4-5):575597, 2011.

67. Philip Moseley, Jay Oswald, and Ted Belytschko. Adaptive atomistic-to-continuum modeling of propagating defects. Int. J. Numer. Methods Eng., 92(10):835-856, 2012.

68. Pierre Kerfriden, Pierre Gosselet, Sondipon Adhikari, and Stephane Pierre-Alain Bordas. Bridging proper orthogonal decomposition methods and augmented newtonkrylov algorithms: an adaptive model order reduction for highly nonlinear mechanical problems. Comput. Methods Appl. Mech. Eng., 200(5):850-866, 2011.

69. Pierre Kerfriden, Jean-Charles Passieux, and Stephane Pierre-Alain Bordas. Local/global model order reduction strategy for the simulation of quasi-brittle fracture. Int. J. Numer. Methods Eng., 89(2):154-179, 2012.

70. Pierre Kerfriden, Olivier Goury, Timon Rabczuk, and Stephane Pierre-Alain Bordas. A partitioned model order reduction approach to rationalise computational expenses in nonlinear fracture mechanics. Comput. Methods Appl. Mech. Eng., 2012.

71. Pierre Kerfriden, Karl Michael Schmidt, Timon Rabczuk, and Stephane Pierre-Alain Bordas. Statistical extraction of process zones and representative subspaces in fracture of random composites. Int. J. Multiscale Comput. Eng., 11(3), 2013.

72. Steven W Cranford and Markus J Buehler. Mechanical properties of graphyne. Carbon, 49(13):4111-4121, 2011.

73. Y. Y. Zhang, Q. X. Pei, and C. M. Wang. Mechanical properties of graphynes under tension: A molecular dynamics study. Appl. Phys. Lett., 101(8):081909, 2012.

74. Adri C. T. van Duin, Siddharth Dasgupta, Francois Lorant, and William A. Goddard. Reaxff: a reactive force field for hydrocarbons. J. Phys. Chem. A, 105(41):9396-9409, 2001.

75. Qing Peng, Wei Ji, and Suvranu De. Mechanical properties of graphyne monolayers: a first-principles study. Phys. Chem. Chem. Phys., 14:13385-13391, 2012.

76. S. Ajori, R. Ansari, and M. Mirnezhad. Mechanical properties of defective -graphyne 
using molecular dynamics simulations. Mater. Sci. Eng., A, 561(0):34-39, 2013.

77. L. D. Pan, L. Z. Zhang, B. Q. Song, S. X. Du, and H. J. Gao. Graphyne- and graphdiyne-based nanoribbons: Density functional theory calculations of electronic structures. Appl. Phys. Lett., 98(17):173102+, 2011.

78. V. Ongun zelik and S. Ciraci. Size dependence in the stabilities and electronic properties of -graphyne and its boron nitride analogue. J. Phys. Chem. C, 117(5):2175-2182, 2013.

79. Jie Tan, Xiujie He, and Mingwen Zhao. First-principles study of hydrogenated graphyne and its family: Stable configurations and electronic structures. Diamond Relat. Mater., 29(0):42 - 47, 2012.

80. Jia-Jia Zheng, Xiang Zhao, Yuliang Zhao, and Xingfa Gao. Two-dimensional carbon compounds derived from graphyne with chemical properties superior to those of graphene. Scientific Reports, 3:-, 2013.

81. Junjie He, Shuang Ying Ma, Pan Zhou, C. X. Zhang, Chaoyu He, and L. Z. Sun. Magnetic properties of single transition-metal atom absorbed graphdiyne and graphyne sheet from dft+u calculations. J. Phys. Chem. C, 116(50):26313-26321, 2012.

82. A.L. Ivanovskii. Graphynes and graphdyines. Prog. Solid State Chem., 41(12):1 - 19, 2013.

83. Michael M. Haley, Stephen C. Brand, and Joshua J. Pak. Carbon networks based on dehydrobenzoannulenes: Synthesis of graphdiyne substructures. Angew. Chem. Int. Ed., 36(8):836-838, 1997.

84. Yang Pei. Mechanical properties of graphdiyne sheet. Physica B, 407(22):4436 4439, 2012.

85. Steven W Cranford and Markus J Buehler. Selective hydrogen purification through graphdiyne under ambient temperature and pressure. Nanoscale, 4(15):4587-4593, 2012.

86. A. Sakhaee-Pour. Elastic properties of single-layered graphene sheet. Solid State Commun., 149(12):91 - 95, 2009.

87. Hui-Juan Cui, Xian-Lei Sheng, Qing-Bo Yan, Qing-Rong Zheng, and Gang Su. Strain-induced dirac cone-like electronic structures and semiconductor-semimetal transition in graphdiyne. Phys. Chem. Chem. Phys., 15:8179-8185, 2013.

88. Jun Kang, Fengmin Wu, and Jingbo Li. Modulating the bandgaps of graphdiyne nanoribbons by transverse electric fields. J. Phys. Condens. Matter, 24(16):165301+, March 2012.

89. Hongxia $\mathrm{Bu}$, Mingwen Zhao, Hongyu Zhang, Xiaopeng Wang, Yan Xi, and Zhenhai Wang. Isoelectronic doping of graphdiyne with boron and nitrogen: Stable configurations and band gap modification. J. Phys. Chem. B, 116(15):3934-3939, 2012.

90. Nobuo Narita, Sumiaki Nagai, Shugo Suzuki, and Kenji Nakao. Optimized geometries and electronic structures of graphyne and its family. Phys. Rev. B, 58:11009-11014, Oct 1998.

91. Qing Peng, Wei Ji, and Suvranu De. Mechanical properties of the hexagonal boron nitride monolayer: Ab initio study. Comput. Mater. Sci., 56:11 - 17, 2012.

92. Xinrui Cao, Yunsong Li, Xuan Cheng, and Ying Zhang. Structural analogues of graphyne family: New types of boron nitride sheets with wide band gap and strong \{UV\} absorption. Chem. Phys. Lett., 502(46):217 - 221, 2011.

93. F. P. Bundy and R. H. Wentorf. Direct Transformation of Hexagonal Boron Nitride to Denser Forms. J. Chem. Phys., 38:1144-1149, 1963.

94. Qing Peng, Amir R. Zamiri, Wei Ji, and Suvranu De. Elastic properties of hybrid graphene/boron nitride monolayer. Acta Mechanica, 223:2591-2596, 2012.

95. Qing Peng, Xiao-Jia Chen, Wei Ji, and Suvranu De. Chemically tuning mechanics 
of graphene by BN. Adv. Eng. Mater., 15:718-727, 2013.

96. Qing Peng, Chen Xiao-Jia, Wei Ji, and Suvranu De. 10.1002/adem.201300033. Adv. Eng. Mater., , 2013.

97. Lei Liu, Y. P. Feng, and Z. X. Shen. Structural and electronic properties of $h$-bn. Phys. Rev. B, 68:104102, Sep 2003.

98. Wei-Qiang Han, Wu Lijun, Zhu Yimei, Kenji Watanabe, and Takashi Taniguchi. Structure of chemically derived mono- and few-atomic-layer boron nitride sheets. Appl. Phys. Lett., 93(22):223103, 2008.

99. Angshuman Nag, Kalyan Raidongia, Kailash P. S. S. Hembram, Ranjan Datta, Umesh V. Waghmare, and C. N. R. Rao. Graphene analogues of bn: Novel synthesis and properties. ACS Nano, 4(3):1539-1544, 2010. PMID: 20128601.

100. Dmitri Golberg, Yoshio Bando, Yang Huang, Takeshi Terao, Masanori Mitome, Chengchun Tang, and Chunyi Zhi. Boron nitride nanotubes and nanosheets. ACS Nano, 4(6):2979-2993, 2010.

101. Qing Peng and Suvranu De. Tunable Band Gaps of Mono-layer Hexagonal BNC Heterostructures. Physica E, 44:1662-1666, APR 2012.

102. J. Kotakoski, C. H. Jin, O. Lehtinen, K. Suenaga, and A. V. Krasheninnikov. Electron knock-on damage in hexagonal boron nitride monolayers. Phys. Rev. B, 82:113404, Sep 2010.

103. Qing Peng, Wei Ji, and Suvranu De. First-principles study of the effects of mechanical strains on the radiation hardness of hexagonal boron nitride monolayers. Nanoscale, 5:695-703, 2013.

104. Susumu Okada. Atomic configurations and energetics of vacancies in hexagonal boron nitride: First-principles total-energy calculations. Phys. Rev. B, 80:161404, Oct 2009.

105. M. Topsakal, E. Aktürk, and S. Ciraci. First-principles study of two- and onedimensional honeycomb structures of boron nitride. Phys. Rev. B, 79:115442, Mar 2009.

106. Kaike Yang, Yuanping Chen, Yuee Xie, X.L. Wei, Tao Ouyang, and Jianxin Zhong. Effect of triangle vacancy on thermal transport in boron nitride nanoribbons. Solid State Commun., 151(6):460 - 464, 2011.

107. Ru-Fen Liu and Ching Cheng. Ab initio studies of possible magnetism in a BN sheet by nonmagnetic impurities and vacancies. Phys. Rev. B, 76:014405, Jul 2007.

108. P. R. Wallace. The band theory of graphite. Phys. Rev., 71:622-634, May 1947.

109. A. K. Geim and K. S. Novoselov. The rise of graphene. Nat. Mater., 6:183-191, 2007.

110. A. K. Geim. Graphene: Status and prospects. Science, 324(5934):1530-1534, 2009.

111. A. H. Castro Neto, F. Guinea, N. M. R. Peres, K. S. Novoselov, and A. K. Geim. The electronic properties of graphene. Rev. Mod. Phys., 81:109-162, Jan 2009.

112. R. Murali. Graphene Nanoelectronics. Springer, first edition, 2012.

113. W. Choi and J. Lee. Graphene: Synthesis and Applications. CRC Press, first edition, 2011.

114. Xiao-Dong Wen, Thomas J. Cahill, and Roald Hoffmann. Exploring group 14 structures: 1d to 2 d to 3d. Chem. Eur. J., 16(22):6555-6566, 2010.

115. Kyozaburo Takeda and Kenji Shiraishi. Theoretical possibility of stage corrugation in si and ge analogs of graphite. Phys. Rev. B, 50:14916-14922, Nov 1994.

116. Gian G. Guzmán-Verri and L. C. Lew Yan Voon. Electronic structure of silicon-based nanostructures. Phys. Rev. B, 76:075131, Aug 2007.

117. S. Cahangirov, M. Topsakal, E. Aktürk, H. Şahin, and S. Ciraci. Two- and onedimensional honeycomb structures of silicon and germanium. Phys. Rev. Lett., 102:236804, Jun 2009.

118. Bernard Aufray, Abdelkader Kara, Sebastien Vizzini, Hamid Oughaddou, Christel 
Leandri, Benedicte Ealet, and Guy Le Lay. Graphene-like silicon nanoribbons on ag(110): A possible formation of silicene. Appl. Phys. Lett., 96(18):183102, 2010.

119. Boubekeur Lalmi, Hamid Oughaddou, Hanna Enriquez, Abdelkader Kara, Sebastien Vizzini, Benidicte Ealet, and Bernard Aufray. Epitaxial growth of a silicene sheet. Appl. Phys. Lett., 97(22):223109, 2010.

120. Paola De Padova, Claudio Quaresima, Bruno Olivieri, Paolo Perfetti, and Guy Le Lay. Strong resistance of silicene nanoribbons towards oxidation. J. Phys. D: Appl. Phys., 44(31):312001, 2011.

121. Chun-Liang Lin, Ryuichi Arafune, Kazuaki Kawahara, Noriyuki Tsukahara, Emi Minamitani, Yousoo Kim, Noriaki Takagi, and Maki Kawai. Structure of silicene grown on ag(111). Appl. Phys. Express, 5(4):045802, 2012.

122. H Jamgotchian, Y Colignon, N Hamzaoui, B Ealet, J Y Hoarau, B Aufray, and J P Bibrian. Growth of silicene layers on ag(111): unexpected effect of the substrate temperature. J. Phys.-Condes. Matter, 24(17):172001, 2012.

123. Baojie Feng, Zijing Ding, Sheng Meng, Yugui Yao, Xiaoyue He, Peng Cheng, Lan Chen, and Kehui Wu. Evidence of silicene in honeycomb structures of silicon on ag(111). Nano Letters, 12(7):3507-3511, 2012.

124. Patrick Vogt, Paola De Padova, Claudio Quaresima, Jose Avila, Emmanouil Frantzeskakis, Maria Carmen Asensio, Andrea Resta, Bénédicte Ealet, and Guy Le Lay. Silicene: Compelling experimental evidence for graphenelike two-dimensional silicon. Phys. Rev. Lett., 108:155501, Apr 2012.

125. Antoine Fleurence, Rainer Friedlein, Taisuke Ozaki, Hiroyuki Kawai, Ying Wang, and Yukiko Yamada-Takamura. Experimental evidence for epitaxial silicene on diboride thin films. Phys. Rev. Lett., 108:245501, Jun 2012.

126. Lei Meng, Yeliang Wang, Lizhi Zhang, Shixuan Du, Rongting Wu, Linfei Li, Yi Zhang, Geng Li, Haitao Zhou, Werner A. Hofer, and Hong-Jun Gao. Buckled silicene formation on ir(111). Nano Letters, 13(2):685-690, 2013.

127. G. Seifert, Th. Köhler, H. M. Urbassek, E. Hernández, and Th. Frauenheim. Tubular structures of silicon. Phys. Rev. B, 63:193409, Apr 2001.

128. H. Şahin, S. Cahangirov, M. Topsakal, E. Bekaroglu, E. Akturk, R. T. Senger, and S. Ciraci. Monolayer honeycomb structures of group-iv elements and iii-v binary compounds: First-principles calculations. Phys. Rev. B, 80:155453, Oct 2009.

129. Nelson Y Dzade, Kingsley O Obodo, Sampson K Adjokatse, Akosa C Ashu, Emmanuel Amankwah, Clement D Atiso, Abdulhakeem A Bello, Emmanuel Igumbor, Stany B Nzabarinda, Joshua T Obodo, Anthony O Ogbuu, Olu Emmanuel Femi, Josephine O Udeigwe, and Umesh V Waghmare. Silicene and transition metal based materials: prediction of a two-dimensional piezomagnet. J. Phys.-Condes. Matter, 22(37):375502, 2010.

130. M. Houssa, G. Pourtois, V. V. Afanas'ev, and A. Stesmans. Can silicon behave like graphene? a first-principles study. Appl. Phys. Lett., 97(11):112106, 2010.

131. Michel Houssa, Geoffrey Pourtois, Marc M. Heyns, Valeri V. Afanas'ev, and Andr Stesmans. Electronic properties of silicene: Insights from first-principles modelling. ECS Transactions, 33(3):185-193, 2010.

132. Zeyuan Ni, Qihang Liu, Kechao Tang, Jiaxin Zheng, Jing Zhou, Rui Qin, Zhengxiang Gao, Dapeng Yu, and Jing Lu. Tunable bandgap in silicene and germanene. Nano Letters, 12(1):113-118, 2012.

133. N. D. Drummond, V. Zólyomi, and V. I. Fal'ko. Electrically tunable band gap in silicene. Phys. Rev. B, 85:075423, Feb 2012.

134. Yunye Liang, Vei Wang, Hiroshi Mizuseki, and Yoshiyuki Kawazoe. Band gap engineering of silicene zigzag nanoribbons with perpendicular electric fields: a theoretical 
study. Appl. Phys. Lett., 24(45):455302, 2012.

135. Junfeng Gao and Jijun Zhao. Initial geometries, interaction mechanism and high stability of silicene on ag(111) surface. Scientific Reports, 2(861), 2012.

136. Hongsheng Liu, Junfeng Gao, and Jijun Zhao. Silicene on substrates: A way to preserve or tune its electronic properties. J. Phys. Chem. C, 117(20):10353-10359, 2013.

137. Lan Chen, Cheng-Cheng Liu, Baojie Feng, Xiaoyue He, Peng Cheng, Zijing Ding, Sheng Meng, Yugui Yao, and Kehui Wu. Evidence for dirac fermions in a honeycomb lattice based on silicon. Phys. Rev. Lett., 109:056804, Aug 2012.

138. Zhi-Xin Guo, Shinnosuke Furuya, Jun ichi Iwata, and Atsushi Oshiyama. Absence of dirac electrons in silicene on ag(111) surfaces. J. Phys. Soc. Jpn., 82(6):063714, 2013.

139. Deepthi Jose and Ayan Datta. Structures and electronic properties of silicene clusters: a promising material for fet and hydrogen storage. Phys. Chem. Chem. Phys., 13:7304-7311, 2011.

140. Y.-P. Wang, J. N. Fry, and H.-P. Cheng. Electron Transport Through Ag-Silicene-Ag Junctions. ArXiv e-prints, May 2013.

141. Guo Wang. Do silicene nanoribbons have high carrier mobilities? Europhys. Lett., 101(2):27005, 2013.

142. TengYong Ng, Jingjie Yeo, and Zishun Liu. Molecular dynamics simulation of the thermal conductivity of shorts strips of graphene and silicene: a comparative study. Int. J. Mech. Mater. Des., 9(2):105-114, 2013.

143. G Le Lay, P De Padova, A Resta, T Bruhn, and P Vogt. Epitaxial silicene: can it be strongly strained? J. Phys. D: Appl. Phys., 45(39):392001, 2012.

144. Qing Peng, XiaoDong Wen, and Suvranu De. Mechanical stabilities of silicene. RSC Adv., 3:13772-13781, 2013.

145. Tim H. Osborn, Amir A. Farajian, Olga V. Pupysheva, Rachel S. Aga, and L.C. Lew Yan Voon. Ab initio simulations of silicene hydrogenation. Chem. Phys. Lett., 511(13):101 - 105, 2011.

146. Chang-wen Zhang and Shi-shen Yan. First-principles study of ferromagnetism in two-dimensional silicene with hydrogenation. J. Phys. Chem. C, 116(6):4163-4166, 2012.

147. Ruge Quhe, Ruixiang Fei, Qihang Liu, Jiaxin Zheng, Hong Li, Chengyong Xu, Zeyuan Ni, Yangyang Wang, Dapeng Yu, Zhengxiang Gao, and Jing Lu. Tunable and sizable band gap in silicene by surface adsorption. Scientific Reports, 2(853), 2012.

148. M. Houssa, E. Scalise, K. Sankaran, G. Pourtois, V. V. Afanas'ev, and A. Stesmans. Electronic properties of hydrogenated silicene and germanene. Appl. Phys. Lett., 98(22):223107, 2011.

149. Yi Ding and Yanli Wang. Electronic structures of silicene fluoride and hydride. Appl. Phys. Lett., 100(8):083102, 2012.

150. Fu-bao Zheng and Chang-wen Zhang. The electronic and magnetic properties of functionalized silicene: a first-principles study. Nanoscale Res. Lett., 7(1):422, 2012.

151. Hengji Zhang, Geunsik Lee, and Kyeongjae Cho. Thermal transport in graphene and effects of vacancy defects. Phys. Rev. B, 84:115460, Sep 2011.

152. Yu-Ling Song, Yan Zhang, Jian-Min Zhang, Dao-Bang Lu, and Ke-Wei Xu. Firstprinciples study of the structural and electronic properties of armchair silicene nanoribbons with vacancies. J. Mol. Struct., 990(13):75 - 78, 2011.

153. Y. C. Cheng, Z. Y. Zhu, and U. Schwingenschlgl. Doped silicene: Evidence of a wide stability range. Europhys. Lett., 95(1):17005, 2011. 
154. Yanli Wang and Yi Ding. Strain-induced self-doping in silicene and germanene from first-principles. Solid State Commun., 155(0):6 - 11, 2013.

155. Fu-bao Zheng, Chang-wen Zhang, Shi-shen Yan, and Feng Li. Novel electronic and magnetic properties in $\mathrm{n}$ or b doped silicene nanoribbons. J. Mater. Chem. C, 1:27352743, 2013.

156. Abdelkader Kara, Hanna Enriquez, Ari P. Seitsonen, L.C. Lew Yan Voon, Sbastien Vizzini, Bernard Aufray, and Hamid Oughaddou. A review on silicene - new candidate for electronics. Surf. Sci. Rep., 67(1):1 - 18, 2012.

157. F. J. DiSalvo, R. Schuall, T. H. Geballe, F. R. Gamble, and J. H. Osiecki. Phys. Rev. Lett., 27:310, 1971.

158. Y. Hamaue and R. Aoki. J. Phys. Soc. Jpn., 55:1327, 1986.

159. E. Furimsky. Catal. Rev., 22:37, 1980.

160. K.K Kam and B.A. Parkinson. J. Phys. Chem., 86:463, 1982.

161. Gmelin Handbook of Inorganic and Organometallic Chemistry. Springer-Verlag, Berlin, 8 edition, 1995.

162. K. S. Novoselov, D. Jiang, F. Schedin, T. J. Booth, V. V. Khotkevich, S. V. Morozov, and A. K. Geim. Proceedings of the National Academy of Sciences, 102:10451, 2005.

163. R. F. Frindt. J. Appl. Phys., 37:1928, 1966.

164. P. Joensen, R.F. Frindt, and S.R. Morrison. Mater. Res. Bull., 21:457, 1986.

165. A. Schumacher, L. Scandella, N. Kruse, and R. Prins. Single-layer MoS2 on mica: studies by means of scanning force microscopy. Surface Science, 289:L595-L598, May 1993.

166. A. Castellanos-Gomez, M. Barkelid, A. M. Goossens, V. E. Calado, H. S. J. van der Zant, and G. A. Steele. Nano Lett., 12:3187, 2012.

167. A. O'Neill, U. Khan, and J. N Coleman. Chem. Mater., 24:2414, 2012.

168. K.-K. Liu, W.J. Zhang, Y.-H. Lee, Y.-C. Lin, M.-T. Chang, C.-Y. Su, C.-S. Chang, H. Li, Y. Shi, H. Zhang, C.-S. Lai, and L.-J. Li. Nano Lett., 12:1538, 2012.

169. K. K. Liu, W. J. Zhang, Y. H. Lee, Y. C. Lin, M. T. Chang, C. Su, C. S. Chang, H. Li, Y. M. Shi, H. Zhang, C. S. Lai, and L. J. Li. Nano Lett., 12:1538, 2012.

170. Y. M. Shi, W. Zhou, A. Y. Lu, W. J. Fang, Y. H. Lee, A. L. Hsu, S. M. Kim, K. K. Kim, H. Y. Yang, L. J. Li, J. C. Idrobo, and J. Kong. Nano Lett., 12:2784, 2012.

171. Y. Liu, H. Nan, X. Wu, W. Pan, W. Wang, J. Bai, W. Zhao, L. Sun, X. Wang, and Z. Ni. ACS Nano, 7:4202, 2013.

172. J. N. Coleman, M. Lotya, A. O’Neill, S. D. Bergin, P. J. King, U. Khan, K. Young, A. Gaucher, S. De, R. J. Smith, I. V. Shvets, S. K. Arora, G. Stanton, H.-Y. Kim, K. Lee, G. T. Kim, G. S. Duesberg, T. Hallam, J. J. Boland, J. J. Wang, J. F. Donegan, J. C. Grunlan, G. Moriarty, A. Shmeliov, R. J. Nicholls, J. M. Perkins, E. M. Grieveson, K. Theuwissen, D. W. McComb, P. D. Nellist, and V. Nicolosi. Two-Dimensional Nanosheets Produced by Liquid Exfoliation of Layered Materials. Science, 331:568, 2011.

173. P. May, U. Khan, J. M. Hughes, and J. N. Coleman. J. Phys. Chem. C, 116:11393, 2012.

174. K.-G. Zhou, N.-N. Mao, H.-X. Wang, Y. Peng, L. Sun, and J. Zhong. Appl. Phys. Lett., 102:071908, 2013.

175. S. Bertolazzi, J. Brivio, and A. Kis. ACS Nano, 5:9703, 2011.

176. A. Castellanos-Gomez, M. Poot, G. A. Steele, H. S. J. van der Zant, N. Agraït, and G. Rubio-Bollinger. Adv. Mater., 24:772775, 2012.

177. T. S. Li. Phys. Rev. B, 85:235407, 2012.

178. X. Tan, J. Wu, K. Zhang, X. Peng, L. Sun, and J. Zhong. Appl. Phys. Lett., 102:071908, 2013. 
179. R. C. Cooper, C. Lee, C. A. Marianetti, X. Wei, J. Hone, and J. W. Kysar. Phys. Rev. B, 87:035423, 2013.

180. Qing Peng and Suvranu De. Outstanding mechanical properties of monolayer mos2 and its application in elastic energy storage. Phys. Chem. Chem. Phys., 2013.

181. D. Jena and A. Konar. Phys. Rev. Lett., 98:136805, 2007.

182. Y. Yoon, K. Ganapathi, and S. Salahuddin. Nano Lett., 11:3768, 2011.

183. D. Lembke and A. Kis. ACS Nano, 6:10070, 2012.

184. J. Pu, Y. Yomogida, K.-K. Liu, L.-J. Li, Y. Iwasa, and T. Takenobu. Nano Lett., 12:4013, 2012.

185. Y. Li, Z. Zhou, S. Zhang, and Z. Chen. J. Am. Chem. Soc., 130:16739, 2008.

186. C. Ataca, H. Sahin, E. Akturk, and S. Ciraci. J. Phys. Chem. C, 115:3934, 2011.

187. S. Helveg, J. V. Lauritsen, E. Lgsgaard, I. Stensgaard, J. K. Nrskov, B.S. Clausen, H. Topse, and F. Besenbacher. Phys. Rev. Lett., 84:951, 2000.

188. J. V. Lauritsen, J. Kibsgaard, S. Helveg, H. Topsoee, B.S. Clausen, E. Laegsgaard, and F. Besenbacher. Nature Nanotech., 2:53, 2007. 